\title{
Bilinear Strichartz's type estimates in Besov spaces with application to inhomogeneous nonlinear biharmonic Schrödinger equation
}

\author{
Xuan Liu, Ting Zhang \\ School of Mathematical Sciences, Zhejiang University, Hangzhou 310027, China
}

\begin{abstract}
In this paper, we consider the well-posedness of the inhomogeneous nonlinear biharmonic Schrödinger equation with spatial inhomogeneity coefficient $K(x)$ behaves like $|x|^{-b}$ for $0<b<\min \left\{\frac{N}{2}, 4\right\}$. We show the local well-posedness in the whole $H^{s}$-subcritical case, with $0<s \leq 2$. The difficulties of this problem come from the singularity of $K(x)$ and the lack of differentiability of the nonlinear term. To resolve this, we derive the bilinear Strichartz's type estimates for the nonlinear biharmonic Schrödinger equations in Besov spaces.

Keywords: Schrödinger equation, Bilinear Strichartz's estimate, Local well-posedness, Besov spaces.
\end{abstract}

\section{Introduction}

In this paper, we consider the Cauchy problem associated to the inhomogeneous biharmonic nonlinear Schrödinger equation

$$
\left\{\begin{array}{l}
i \partial_{t} u+\Delta^{2} u+\mu \Delta u+K(x) f(u)=0, \quad t \in \mathbb{R}, x \in \mathbb{R}^{N} \\
u(0, x)=\varphi(x)
\end{array}\right.
$$

where $N \geq 1, \mu=-1$ or $0, u: \mathbb{R} \times \mathbb{R}^{N} \rightarrow \mathbb{C}$ is a complex-valued function, $K(x), f(u)$ are the inhomogeneity coefficient and the nonlinear term, respectively. Note that if $\mu=0$ and $K(x)=\lambda|x|^{-b}, f(u)=|u|^{\alpha} u$ with $\lambda \in \mathbb{C}, 0<b<\min \left\{4, \frac{N}{2}\right\}, \alpha>0$, the equation (1.1) is invariant under the scaling, $u_{k}(t, x)=k^{\frac{4-b}{\alpha}} u\left(k^{4} t, k x\right), k>0$. This means if $u$ is a solution of (1.1) with the initial datum $\phi$, so is $u_{k}$ with the initial datum $\phi_{k}=k^{\frac{4-b}{\alpha}} \phi(k x)$. Computing the homogeneous Sobolev norm, we get

$$
\left\|\phi_{k}\right\|_{\dot{H}^{s}}=k^{s-\frac{N}{2}+\frac{4-b}{\alpha}}\|\phi\|_{\dot{H}^{s}} .
$$

The Sobolev index which leaves the scaling symmetry invariant is called the critical index and is defined as $s_{c}=\frac{N}{2}-\frac{4-b}{\alpha}$. If $s_{c}=s$ (equivalently $\alpha=\frac{8-2 b}{N-2 s}$ ), the Cauchy problem (1.1) is known as $H^{s}$-critical; if $s_{c}>s$ (equivalently $\left.0<\alpha,(N-2 s) \alpha<8-2 b\right)$, it is called $H^{s}$-subcritical. The limiting case $b=0$ (classical biharmonic nonlinear Schrödinger equation, also called the fourth-order Schrödinger equation) has been introduced by Karpman [22], and Karpman-Shagalov [23] to take into account the role of small fourth-order dispersion terms in the propagation of intense laser beams in a bulk medium with Kerr nonlinearity. Since then, the study of nonlinear fourth-order Schrödinger equation has 
been attracted a lot of interest in the past decade. See [7, 16, 20, 27, 28, 30, 31] and references cited therein.

The equation in (1.1) has a counterpart for the Laplacian operator, namely, the inhomogeneous nonlinear Schrödinger equation

$$
i \partial_{t} u+\Delta u+K(x)|u|^{\alpha} u=0
$$

In Gill [14] and Liu-Tripathi [25, it was suggested that stable high power propagation can be achieved in a plasma by sending a preliminary laser beam that creates a channel with a reduced electron density, and thus reduces the nonlinearity inside the channel. In this case, the beam propagation can be modeled by the inhomogeneous nonlinear Schrödinger equation (1.2). In addition, Fibich and Wang [1] investigated (1.2) for $K(\varepsilon|x|)$ with $\varepsilon$ small and $K \in C^{4}\left(\mathbb{R}^{N}\right) \cap L^{\infty}\left(\mathbb{R}^{N}\right)$, where the solution $u$ is the electric field in laser and optics, and $K(x)$ is proportional to the electron density with a small parameter $\varepsilon>0$ (see also [18). For other interesting Physical applications of (1.2), we refer to [2, 3, 4, 21, 26, 33, 34 and the references therein.

Let us first review some known well-posedness results for (1.2). We shall assume $K(x)=\lambda|x|^{-b}$ with $\lambda \in \mathbb{R}, 0<b<2$ to make the review shorter. Genoud and Stuart [13] first showed the local well-posedness in $H^{1}\left(\mathbb{R}^{N}\right)$ for $0<b<\min \{2, N\}$ and $0<\alpha$, $(N-2) \alpha<4-2 b$ by using the abstract argument of Cazenave [6], which does not use Strichartz's estimates. In this case, Genoud 12 and Farah 10 also showed how small should be the initial data to have global well-posedness, respectively, in the spirit of Weinstein [35] and Holmer-Roudenko [17] for the classical case $b=0$. Recently, using Strichartz's estimate and the contraction mapping argument, Guzmán [15] showed the local well-posedness of (1.2) for $0<\alpha,(N-2) \alpha<4-2 b$, but under the restrictions: $b<\frac{N}{3}$ if $N \leq 3$. This restriction is a bit improved by Dinh [8] in dimension $N=3$, for $0<b<\frac{3}{2}$ but for more restricted values $\alpha<\frac{6-4 b}{2 b-1}$. Although these results are a bit weak on the range of $b$ compared with the result of Genoud-Stuart, they provide more information on the solution due to the Strichartz's estimates. In particular, one can know that the local solutions belong to $L_{l o c}^{p}\left(\left[0, T_{\max }\right), L^{q}\left(\mathbb{R}^{N}\right)\right)$ for any Schrödinger admissible pair $(p, q)$. In general, such property plays an important role in studying other interesting problems, for instance, scattering and blow up. Finally, we review the well-posedness of (1.2) in $H^{s}$. Defining

$$
\hat{2}:= \begin{cases}\min \left\{2,1+\frac{n-2 s}{2}\right\}, & n \geq 3, \\ n-s, & n=1,2,\end{cases}
$$

it was proved in [1] that, for $N \geq 1,0<b<\hat{2}$, and $0 \leq s<\frac{N}{2}, 0<\alpha<\frac{4-2 b}{N-2 s}$ or $\frac{N}{2} \leq s<\min \left\{N, \frac{N}{2}+1\right\}, 0<\alpha<\infty$, the Cauchy problem (1.2) is local wellposed in $H^{s}\left(\mathbb{R}^{N}\right)$. Moreover, it was proved in [24] that (1.2) is local well-posed in a weighted Sobolev space for $N \geq 3,0<s<\frac{1}{3}, \max \left\{\frac{26-3 N}{12}, \frac{12 s+4 N s-8 s^{2}}{N+4 s}\right\}<b<2$ and $\max \left\{0, \frac{10 s-2 \alpha}{N-6 s}\right\}<\alpha \leq \frac{4-2 b}{N-2 s}$.

In this paper we are interested in studying the well-posedness for (1.1) in $H^{s}\left(\mathbb{R}^{N}\right)$, with $0<s \leq 2$. This problem was firstly studied by C.M. Guzmán and A. Pastor [16] for $K(x)=\lambda|x|^{-b}, f(u)=|u|^{\alpha} u, \lambda \in \mathbb{R}$. They proved the local-well posedness in $H^{2}$ for $N \geq 3,0<b<\min \left\{\frac{N}{2}, 4\right\}, \max \left\{0, \frac{2(1-b)}{N}\right\}<\alpha,(N-4) \alpha<8-2 b$. Also, they proved global well-posedness in the mass-subcritical and mass-critical cases in $H^{2}$, that 
is, $\min \left\{\frac{2(1-b)}{N}, 0\right\}<\alpha \leq \frac{4-b}{N}$. Afterwards, Cardoso-Guzman-Pastor [5] established the local well-posedness in $\dot{H}^{s} \cap \dot{H}^{2}$ with $N \geq 5,0<s<2,0<b<\min \left\{\frac{N}{2}, 4\right\}$ and $\max \left\{\frac{8-2 b}{N}, 1\right\}<\alpha<\frac{8-2 b}{N-4}$. Note that [16, 5] does not treat the low dimensions cases and there is a lower bound for the parameter $\alpha$. The restrictions of dimensions and index $\alpha$ are due to the singularity of inhomogeneity coefficient and the lack of differentiability of the nonlinear term. To resolve this, we derive the following bilinear Strichartz's type estimates for nonlinear biharmonic Schrödinger equations in Besov spaces, by which we can replace the spatial derivative of order $s$ with the fractional order time derivative of order $s / 4$. For the definition of the biharmonic admissible set $\Lambda_{b}$, we refer to Section 2 ,

Proposition 1.1. Let $N \geq 1, \mu=0$ or $-1,0<s \leq 2,(q, r),\left(\gamma_{0}, \rho_{0}\right),(\gamma, \rho),\left(\gamma_{1}, \rho_{1}\right) \in \Lambda_{b}$ be four biharmonic admissible pairs, and $1 \leq \bar{q}, \bar{r} \leq 2$ with $\frac{4}{\bar{q}}-N\left(\frac{1}{2}-\frac{1}{\bar{r}}\right)=4-s$. Then for any $\varphi \in H^{s}\left(\mathbb{R}^{N}\right)$ and $f \in B_{\gamma_{0}^{\prime}, 2}^{s / 4} L^{\rho_{0}^{\prime}} \cap L^{\bar{q}} L^{\bar{r}}$, we have $e^{i t\left(\Delta^{2}+\mu \Delta\right)} \varphi, G f \in C\left(\mathbb{R}, H^{s}\right)$, where

$$
(G f)(t):=\int_{0}^{t} e^{i(t-s)\left(\Delta^{2}+\mu \Delta\right)} f(s) d s .
$$

Moreover, the following inequalities hold:

$$
\left\|e^{i t\left(\Delta^{2}+\mu \Delta\right)} \varphi\right\|_{L^{q} B_{r, 2}^{s} \cap B_{q, 2}^{s / 4} L^{r}} \lesssim\|\varphi\|_{H^{s}}
$$

and

$$
\begin{aligned}
\|G(f g)\|_{L^{q} B_{r, 2}^{s} \cap B_{q, 2}^{s / 4} L^{r}} \lesssim & \left(\int_{-\infty}^{\infty}\left(|\tau|^{-s / 4}\|g(t-\tau)(f(t-\tau)-f(t))\|_{L^{\gamma_{0}^{\prime}} L^{\rho_{0}^{\prime}}}\right)^{2} \frac{d \tau}{|\tau|}\right)^{1 / 2} \\
& +\left(\int_{-\infty}^{\infty}\left(|\tau|^{-s / 4}\|(g(t-\tau)-g(t)) f(t)\|_{L^{\gamma_{1}^{\prime}} L^{\rho_{1}^{\prime}}}\right)^{2} \frac{d \tau}{|\tau|}\right)^{1 / 2} \\
& +\|f g\|_{L^{\gamma^{\prime} L^{\rho^{\prime}} \cap L^{q} L^{\bar{r}}}}
\end{aligned}
$$

Remark 1.2. Proposition 1.1 is a refinement of the following inequality previously obtained by Nakamura and Wada for classical Schrödinger equation in 29]:

$$
\|G f\|_{L^{q} B_{r, 2}^{s} \cap B_{q, 2}^{s / 2} L^{r}} \lesssim\|f\|_{B_{\gamma^{\prime}, 2}^{s / 2} \rho^{\rho^{\prime}}}+\|f\|_{L^{\bar{q}} L^{\bar{r}}}
$$

where $0<s<2,(q, r),(\gamma, \rho)$ are two classical Schrödinger admissible pairs and $1 \leq \bar{q}, \bar{r} \leq$ 2 with $\frac{2}{\bar{q}}-N\left(\frac{1}{2}-\frac{1}{\bar{r}}\right)=2-s$. The most important advantage of our new estimates (1.5) is that we can choose the admissible pairs $\left(\gamma_{0}, \rho_{0}\right),\left(\gamma_{1}, \rho_{1}\right)$ independently when the difference falls on different functions. Thus we can choose the index more flexible when establishing the nonlinear estimates needed for the contraction argument. This can be an advantage when we consider not only pure power but also more complicated nonlinear terms.

Using the bilinear Strichartz's type estimate, the detailed technique of choosing indices (see Lemmas 4.1 4.10) and applying various embeddings in nonlinear estimates, we can establish the $H^{s}$ local well-posedness for (1.1) in the whole $H^{s}$ subcritical case, with $0<s \leq 2$. Before stating our results, we give the following notation.

Definition 1.3. Let $\alpha>0, f \in C^{1}(\mathbb{C}, \mathbb{C})$ in the real sense. We say that $f$ belongs to the class $\alpha$ if it satisfies $f(0)=0$ and

$$
\left|f^{\prime}\left(z_{1}\right)-f^{\prime}\left(z_{2}\right)\right| \lesssim\left(\left|z_{1}\right|^{\alpha-1}+\left|z_{2}\right|^{\alpha-1}\right)\left|z_{1}-z_{2}\right|, \quad \forall z_{1}, z_{2} \in \mathbb{C} .
$$


Remark 1.4. We note that the power type nonlinearities $f(u)=\lambda|u|^{\alpha} u$ and $f(u)=$ $\lambda|u|^{\alpha+1}$ with $\lambda \in \mathbb{C}, \alpha>0$ are in the class $\mathcal{C}(\alpha)$. Moreover, for any $\alpha>0$ and $f \in \mathcal{C}(\alpha)$, it is easy to check that the following inequality holds for any $u, v \in \mathbb{C}$,

$$
|f(u)-f(v)| \lesssim\left(|u|^{\alpha}+|v|^{\alpha}\right)|u-v| .
$$

Now we are ready to state our main result.

Theorem 1.5. Let $N \geq 1, \mu=0$ or $-1, \beta>\max \left\{2, \frac{N}{4}\right\}, 0<s \leq 2,0<\alpha,(N-2 s) \alpha<$ $8-\frac{2 N}{\beta}, f \in \mathcal{C}(\alpha)$, and $K(x) \in L^{\infty}\left(\mathbb{R}^{N}\right)+L^{\beta}\left(\mathbb{R}^{N}\right)$. Given $\varphi \in H^{s}\left(\mathbb{R}^{N}\right)$, there exists $T_{\max }(\varphi) \in(0, \infty]$ and a unique maximum solution $u \in C\left(\left[0, T_{\max }(\varphi)\right), H^{s}\right) \bigcap_{(q, r) \in \Lambda_{b}} L^{q}$ $\left.\left(\left[0, T_{\max }(\varphi)\right), B_{r, 2}^{s}\right)\right)$ to the Cauchy problem (1.1), with the following blowup alternative holds:

$$
\lim _{t \uparrow T_{\max }}\|u(t)\|_{H^{s}}=\infty, \quad \text { if } T_{\max }(\varphi)<\infty .
$$

Moreover, if $\varphi_{n} \rightarrow \varphi$ in $H^{s}\left(\mathbb{R}^{N}\right)$ and $u_{n}$ denotes the solution of (1.1) with the initial value $\varphi_{n}$, then $u_{n} \rightarrow u$ in $C\left([0, A], H^{s}\left(\mathbb{R}^{N}\right)\right)$ for any $0<A<T_{\max }(\varphi)$.

The argument used to derive Theorem 1.5 can also be applied to the classical inhomogeneous nonlinear Schrödinger equation (1.2). More precisely, we can establish the bilinear Strichartz's type estimate for (1.2) in the spirit of Proposition 1.1. Then we choose the index as in Lemmas 4.14 .10 to establish a series of estimates needed in the contraction argument. Therefore this improves the above mentioned results in [1, 24] on the validity of $\alpha$ and $b$ in the case $0<s<1$.

If $K(x)=\lambda|x|^{-b}$ and $f(u)=|u|^{\alpha} u$, with $\lambda \in \mathbb{C}, b>0, \alpha>0$, we then have the following result, which removes the lower bound $\alpha>\frac{2(1-b)}{N}$ made in [16].

Corollary 1.6. Let $N \geq 1,0<b<\min \left\{\frac{N}{2}, 4\right\}, 0<\alpha,(N-4) \alpha<8-2 b, \lambda \in \mathbb{C}$ and $\mu=-1$ or 0 . Given $\varphi \in H^{2}\left(\mathbb{R}^{N}\right)$, there exists $T_{\max }(\varphi) \in(0, \infty]$ and a unique maximum solution $\left.u \in C\left(\left[0, T_{\max }(\varphi)\right), H^{2}\right) \bigcap_{(q, r) \in \Lambda_{b}} L^{q}\left(\left[0, T_{\max }(\varphi)\right), B_{r, 2}^{2}\right)\right)$ to the Cauchy problem

$$
\left\{\begin{array}{l}
i \partial_{t} u+\left(\Delta^{2}+\mu \Delta\right) u+\lambda|x|^{-b}|u|^{\alpha} u=0, \\
u(0, x)=\varphi(x)
\end{array}\right.
$$

with the following blowup alternative holds:

$$
\lim _{t \uparrow T_{\max }}\|u(t)\|_{H^{2}}=\infty, \quad \text { if } T_{\max }(\varphi)<\infty .
$$

Moreover, the continuous dependence upon the initial data holds.

If $K(x)=\lambda$ with $\lambda \in \mathbb{C}$ and $f(u)=|u|^{\alpha} u$, we then have the following result for fourth-order Schrödinger equation.

Corollary 1.7. Let $N \geq 1,0<s \leq 2,0<\alpha,(N-2 s) \alpha<8, \lambda \in \mathbb{C}$ and $\mu=-1$ or 0 . Given $\varphi \in H^{s}\left(\mathbb{R}^{N}\right)$, there exists $T_{\max }(\varphi) \in(0, \infty]$ and a unique maximum solution $\left.u \in C\left(\left[0, T_{\max }(\varphi)\right), H^{s}\right) \bigcap_{(q, r) \in \Lambda_{b}} L^{q}\left(\left[0, T_{\max }(\varphi)\right), B_{r, 2}^{s}\right)\right)$ to the Cauchy problem

$$
\left\{\begin{array}{l}
i \partial_{t} u+\left(\Delta^{2}+\mu \Delta\right) u+\lambda|u|^{\alpha} u=0 \\
u(0, x)=\varphi(x)
\end{array}\right.
$$

with the following blowup alternative holds:

$$
\lim _{t \uparrow T_{\max }}\|u(t)\|_{H^{s}}=\infty, \quad \text { if } T_{\max }(\varphi)<\infty .
$$

Moreover, the continuous dependence upon the initial data holds. 
Remark 1.8. Corollary 1.7 improves the corresponding results of [7, 9] in the case $0<$ $s \leq 2$. In [7, there is a additional assumption $s \leq[\alpha]([\alpha]$ denotes the largest integer less than or equal to $\alpha)$ for the parameter $\alpha$; and in [ $\left[\right.$, the continuous dependence $\left(u_{n} \rightarrow u\right.$ in $C\left([0, T], H^{s-\varepsilon}\right)$ with $\left.\varepsilon>0\right)$ is weaker than the expected one.

In the sequel, we establish global existence results in energy space in the $L^{2}$-subcritical regime. We assume that $K(x)$ is a real-valued function, which will be used to establish the conservation of the mass.

Theorem 1.9. Let $N \geq 1, \mu=0$ or $-1, \beta>\max \left\{2, \frac{N}{4}\right\}, 0<\alpha \leq \frac{8}{N}-\frac{2}{\beta}, K(x) \in$ $L^{\infty}\left(\mathbb{R}^{N}\right)+L^{\beta}\left(\mathbb{R}^{N}\right)$ be a real-valued function, and $f \in \mathcal{C}(\alpha)$ that satisfies

(i) $f(a) \in \mathbb{R}$ for all $a \geq 0$;

(ii) $f(u)=\frac{u}{|u|} f(|u|)$ for all $u \in \mathbb{C} \backslash\{0\}$;

Then the local solution obtained in Theorem 1.5 with the initial datum $\varphi$ can be extended globally-in-time if one of the following alternatives holds:

(i) $0<\alpha<\frac{8}{N}-\frac{2}{\beta}$, or

(ii) $\alpha=\frac{8}{N}-\frac{2}{\beta}$ and $\|\varphi\|_{L^{2}}$ sufficiently small.

If $K(x)=\lambda|x|^{-b}$ and $f(u)=|u|^{\alpha} u$ with $\lambda \in \mathbb{R}$, we then have the following result, which removes the lower bound $\alpha>\frac{2(1-b)}{N}$ made in [16].

Corollary 1.10. Let $N \geq 1,0<b<\min \left\{\frac{N}{2}, 4\right\}, 0<\alpha \leq \frac{8-2 b}{N}$, and $K(x)=\lambda|x|^{-b}$, $f(u)=|u|^{\alpha} u, \lambda \in \mathbb{R}$. Then the local solution obtained in Corollary 1.6 with the initial datum $\varphi$ can be extended globally-in-time if one of the following alternatives holds:

(i) $0<\alpha<\frac{8-2 b}{N}$, or

(ii) $\alpha=\frac{8-2 b}{N}$ and $\|\varphi\|_{L^{2}}$ sufficiently small.

When $K(x)$ is a complex-valued function, the solutions to (1.1) may blow up in finite time. In fact, for any given compact set $M \subset \mathbb{R}^{N}$, it was proved in [19] that, for $K(x)=$ $\lambda, f(u)=|u|^{\alpha} u$ with $\operatorname{Im} \lambda>0,0<\alpha<\frac{8}{N-8}$, there exists a class of solutions to (1.1), which blows up exactly on $M$.

The rest of the paper is organized as follows. In Section 2 we introduce some notations and give a review of the biharmonic Strichartz's estimates. In Section 3, we establish the bilinear Strichartz's type estimate. In Section 4 , we establish the nonlinear estimates that are needed in the contraction argument. In Section 5 , we give the proof of Theorem 1.5 and Theorem 1.9 .

\section{Preliminary}

If $X, Y$ are nonnegative quantities, we sometimes use $X \lesssim Y$ to denote the estimate $X \leq C Y$ for some positive constant $C$. Pairs of conjugate indices are written as $p$ and $p^{\prime}$, where $1 \leq p \leq \infty$ and $\frac{1}{p}+\frac{1}{p^{\prime}}=1$. We use $L^{p}\left(\mathbb{R}^{N}\right)$ to denote the usual Lebesgue space and $L^{\gamma}\left(I, L^{\rho}\left(\mathbb{R}^{N}\right)\right)$ to denote the space-time Lebesgue spaces with the norm

$$
\|f\|_{L^{\gamma}\left(I, L^{\rho}\left(\mathbb{R}^{N}\right)\right)}:=\left(\int_{I}\|f\|_{L_{x}^{\rho}}^{\gamma} d t\right)^{1 / \gamma}
$$

for any time slab $I \subset \mathbb{R}$, with the usual modification when either $\gamma$ or $\rho$ is infinity. We also define the Fourier transform on $\mathbb{R}, \mathbb{R}^{N}$ and $\mathbb{R}^{1+N}$ by

$$
\hat{f}(\tau)=\int_{\mathbb{R}} f(t) e^{-i t \tau} d t, \quad \tau \in \mathbb{R}
$$




$$
\begin{array}{ll}
\hat{f}(\xi)=\int_{\mathbb{R}^{N}} f(x) e^{-i x \cdot \xi} d x, & \xi \in \mathbb{R}^{N} ; \\
\tilde{f}(\tau, \xi)=\int_{\mathbb{R}^{1+N}} f(t, x) e^{-i x \cdot \xi-i t \tau} d x d t, & (\tau, \xi) \in \mathbb{R} \times \mathbb{R}^{N},
\end{array}
$$

respectively.

Next, we review the definition of Besov spaces. Let $\phi$ be a smooth function whose Fourier transform $\hat{\phi}$ is a non-negative even function which satisfies supp $\hat{\phi} \subset\{\tau \in \mathbb{R}, 1 / 2 \leq$ $|\tau| \leq 2\}$ and $\sum_{k=-\infty}^{\infty} \hat{\phi}\left(\tau / 2^{k}\right)=1$ for any $\tau \neq 0$. For $k \in \mathbb{Z}$, we put $\hat{\phi}_{k}(\cdot)=\hat{\phi}\left(\cdot / 2^{k}\right)$ and $\psi=\sum_{j=-\infty}^{0} \phi_{j}$. Moreover, we define $\chi_{k}=\sum_{k-2}^{k+2} \phi_{j}$ for $k \geq 1$ and $\chi_{0}=\psi+\phi_{1}+\phi_{2}$. For $s \in \mathbb{R}$ and $1 \leq p, q \leq \infty$, we define the Besov space

$$
B_{p, q}^{s}\left(\mathbb{R}^{N}\right)=\left\{u \in \mathcal{S}^{\prime}\left(\mathbb{R}^{N}\right),\|u\|_{B_{p, q}^{s}\left(\mathbb{R}^{N}\right)}<\infty\right\}
$$

where $\mathcal{S}^{\prime}\left(\mathbb{R}^{N}\right)$ is the space of tempered distributions on $\mathbb{R}^{N}$, and

$$
\|u\|_{B_{p, q}^{s}\left(\mathbb{R}^{N}\right)}=\left\|\psi *_{x} u\right\|_{L^{p}\left(\mathbb{R}^{N}\right)}+ \begin{cases}\left\{\sum_{k \geq 1}\left(2^{s k}\left\|\phi_{k} *_{x} u\right\|_{L^{p}\left(\mathbb{R}^{N}\right)}\right)^{q}\right\}^{1 / q}, & q<\infty \\ \sup _{k \geq 1} 2^{s k}\left\|\phi_{k} *_{x} u\right\|_{L^{p}\left(\mathbb{R}^{N}\right)}, & q=\infty\end{cases}
$$

where $*_{x}$ denotes the convolution with respect to the variables in $\mathbb{R}^{N}$. Here we use $\phi_{k} *_{x} u$ to denote $\phi_{k}(|\cdot|) *_{x} u$. We also define $\chi_{k} *_{x} u, \psi *_{x} u, \chi_{0} *_{x} u$ similarly. This is an abuse of symbol, but no confusion is likely to arise.

For $1 \leq q, \alpha \leq \infty$ and a Banach space $V$, we denote the vector-valued Lebesgue space for functions on $\mathbb{R}$ to $V$ by $L^{q}(\mathbb{R}, V)$. Then we define the vector-valued Sobolev space

$$
H^{1, q}(\mathbb{R}, V)=\left\{u: u \in L^{q}(\mathbb{R}, V), \partial_{t} u \in L^{q}(\mathbb{R}, V)\right\} .
$$

Finally, we define the Besov space of vector-valued functions. Let $\theta \in \mathbb{R}, 1 \leq q, \alpha \leq \infty$ and $V$ be a Banach space. We put

$$
B_{q, \alpha}^{\theta}(\mathbb{R}, V)=\left\{u \in \mathcal{S}^{\prime}(\mathbb{R}, V) ;\|u\|_{B_{q, \alpha}^{\theta}(\mathbb{R}, V)}<\infty\right\},
$$

where

$$
\|u\|_{B_{q, \alpha}^{\theta}(\mathbb{R}, V)}=\left\|\psi *_{t} u\right\|_{L^{q}(\mathbb{R}, V)}+\left\{\sum_{k \geq 1}\left(2^{\theta k}\left\|\phi_{k} *_{t} u\right\|_{L^{q}(\mathbb{R}, V)}\right)^{\alpha}\right\}^{1 / \alpha}
$$

with trivial modification if $\alpha=\infty$. Here $*_{t}$ denotes the convolution in $\mathbb{R}$. Moreover, it is well-known that the norm (2.1) has the following equivalence

$$
\|u\|_{B_{q, \alpha}^{\theta}(\mathbb{R}, V)} \approx\|u\|_{L^{q}(\mathbb{R}, V)}+\left(\int_{-\infty}^{\infty}\left(\tau^{-s}\|u(t)-u(t-\tau)\|_{L^{q}(\mathbb{R}, V)}\right)^{q} \frac{d \tau}{|\tau|}\right)^{1 / q} .
$$

Following standard notations, we introduce Schrödinger admissible pair as well as the corresponding Strichartz's estimate for the biharmonic Schrödinger equation.

Definition 2.1. A pair of Lebesgue space exponents $(\gamma, \rho)$ is called biharmonic Schrödinger admissible for the equation (1.1) if $(\gamma, \rho) \in \Lambda_{b}$ where

$$
\Lambda_{b}=\left\{(\gamma, \rho): 2 \leq \gamma, \rho \leq \infty, \frac{4}{\gamma}+\frac{N}{\rho}=\frac{N}{2},(\gamma, \rho, N) \neq(2, \infty, 4)\right\} .
$$


Lemma 2.2 (Strichartz's estimate for BNLS). Suppose that $(\gamma, \rho),(a, b) \in \Lambda_{b}$ are two biharmonic admissible pairs, and $\mu=0$ or -1 . Then for any $u \in L^{2}\left(\mathbb{R}^{N}\right)$ and $h \in$ $L^{a^{\prime}}\left(\mathbb{R}, L^{b^{\prime}}\left(\mathbb{R}^{N}\right)\right)$, we have

$$
\begin{gathered}
\left\|e^{i t\left(\Delta^{2}+\mu \Delta\right)} u\right\|_{L^{\gamma} L^{\rho}} \lesssim\|u\|_{L^{2}}, \\
\left\|\int_{0}^{t} e^{i(t-s)\left(\Delta^{2}+\mu \Delta\right)} h(s) d s\right\|_{L^{\gamma} L^{\rho}} \lesssim\|h\|_{L^{a^{\prime}} L^{b^{\prime}}}, \\
\left\|\int_{t}^{\infty} e^{i(t-s)\left(\Delta^{2}+\mu \Delta\right)} h(s) d s\right\|_{L^{\gamma} L^{\rho}} \lesssim\|h\|_{L^{a^{\prime} L^{b^{\prime}}}} .
\end{gathered}
$$

Proof. The estimates (2.2) and (2.4) are proved in [30]; and the proof of (2.4) is follows from an obvious adaptation of Corollary 2.3.6 in [6].

In this paper, we omit the integral domain for simplicity unless noted otherwise. For example, we write $L^{q} L^{r}=L^{q}\left(\mathbb{R}, L^{r}\left(\mathbb{R}^{N}\right)\right), L^{q} B_{r, 2}^{s}=L^{q}\left(\mathbb{R}, B_{r, 2}^{s}\left(\mathbb{R}^{N}\right)\right)$ and $B_{q, 2}^{\theta} L^{r}=$ $B_{q, 2}^{\theta}\left(\mathbb{R}, L^{r}\left(\mathbb{R}^{N}\right)\right)$ etc.

\section{Bilinear Strichartz's type estimate}

In this section we prove Proposition 1.1. First, we prepare several lemmas. We assume the functions $\phi, \chi_{0}, \psi, \phi_{j}, \chi_{j}$ are defined in Section 2

Lemma 3.1 (Lemma 3.1 in [20]). Assume $N \geq 1, \mu=-1$ or 0 , and $K_{j}(t, x)(j \geq 1)$ : $\mathbb{R} \times \mathbb{R}^{N} \rightarrow \mathbb{C}$ are defined by

$$
K_{j}(t, x)=\frac{1}{(2 \pi)^{1+N}} \int e^{i t \tau+i x \cdot \xi} \frac{\hat{\phi}_{j}\left(|\xi|^{4}-\mu|\xi|^{2}\right)\left(1-\hat{\chi}_{j}(\tau)\right)}{i\left(\tau-|\xi|^{4}+\mu|\xi|^{2}\right)} d \tau d \xi
$$

Then for any $0<s<4,1 \leq q \leq \infty, 1 \leq r \leq \infty$ with $\frac{4}{q}-N\left(1-\frac{1}{r}\right)=s$, we have

$$
\left\|K_{j}\right\|_{L^{q} L^{r}} \leq C 2^{-j s / 4},
$$

where the constant $C$ is independent of $j \geq 1$.

Lemma 3.2 (Lemma 3.3 in [20]). Let $s \in \mathbb{R}, 1 \leq p, q \leq \infty$, then the norm defined by

$$
\begin{aligned}
& \|u\|_{\widetilde{B}_{p, q}^{s}\left(\mathbb{R}^{n}\right)}:=\left\|\left(\mathcal{F}_{\xi}^{-1}\left(\widehat{\psi}\left(|\xi|^{4}-\mu|\xi|^{2}\right)\right)\right) *_{x} u\right\|_{L^{p}\left(\mathbb{R}^{n}\right)} \\
& +\left\{\begin{array}{l}
\left\{\sum_{j \geq 1}\left(2^{s j / 4}\left\|\left(\mathcal{F}_{\xi}^{-1}\left(\widehat{\phi}\left(\left(|\xi|^{4}-\mu|\xi|^{2}\right) / 2^{j}\right)\right)\right) *_{x} u\right\|_{L^{p}\left(\mathbb{R}^{n}\right)}\right)^{q}\right\}^{1 / q}, \text { if } q<\infty, \\
\sup _{j \geq 1} 2^{s j / 4}\left\|\left(\mathcal{F}_{\xi}^{-1}\left(\widehat{\phi}\left(\left(|\xi|^{4}-\mu|\xi|^{2}\right) / 2^{j}\right)\right)\right) *_{x} u\right\|_{L^{p}\left(\mathbb{R}^{n}\right)}, \text { if } q=\infty,
\end{array}\right.
\end{aligned}
$$

is equivalent to the norm $\|u\|_{B_{p, q}^{s}}\left(\mathbb{R}^{N}\right)$ for any function $u$.

In the rest of this section, we use the natation $\phi_{j / 4}=\mathcal{F}_{\xi}^{-1}\left(\hat{\phi}_{j}\left(|\xi|^{4}-\mu|\xi|^{2}\right)\right)$. This is an abuse of symbol, but no confusion is likely to arise. Under this notation, we obtain the following equivalence from Lemma 3.2

$$
\|u\|_{B_{p, q}^{s}} \approx\left\|\left(\mathcal{F}_{\xi}^{-1}\left(\widehat{\psi}\left(|\xi|^{4}-\mu|\xi|^{2}\right)\right)\right) *_{x} u\right\|_{L^{p}}+\left(\sum_{j=1}^{\infty}\left(2^{s j / 4}\left\|\phi_{j / 4} *_{x} u\right\|_{L^{p}}\right)^{q}\right)^{1 / q}
$$

with trivial modification if $q=\infty$. 
Lemma 3.3 (Lemma 2.1 in [32]). Suppose that $N \geq 1,1 \leq \gamma<\infty, 1 \leq \rho \leq \infty, 0<\theta<1$, and $1 \leq q \leq \infty$, then we have

$$
\left(L^{\gamma}\left(\mathbb{R}, L^{\rho}\left(\mathbb{R}^{N}\right)\right), H^{1, \gamma}\left(\mathbb{R}, L^{\rho}\left(\mathbb{R}^{N}\right)\right)\right)_{\theta, q}=B_{\gamma, q}^{\theta}\left(\mathbb{R}, L^{\rho}\left(\mathbb{R}^{N}\right)\right) .
$$

Proof of Proposition 1.1. The continuity of $G f$ and $e^{i t\left(\Delta^{2}+\mu \Delta\right)} \varphi$ in time follows from density argument. We now prove the inequality (1.4). Using the similar argument as that used in the proof of Corollary 2.3.9 in [6], we obtain the following estimates and omit the details:

$$
\begin{gathered}
\left\|e^{i t\left(\Delta^{2}+\mu \Delta\right)} \phi\right\|_{L^{q}\left(\mathbb{R}, B_{r, 2}^{\tilde{s}}\right)} \lesssim\|\phi\|_{H^{\tilde{s}},} \\
\left\|\frac{d}{d t}\left(e^{i t\left(\Delta^{2}+\mu \Delta\right)} \phi\right)\right\|_{L^{q}\left(\mathbb{R}, B_{r, 2}^{\tilde{s}}\right)} \lesssim\|\phi\|_{H^{\tilde{s}+4}},
\end{gathered}
$$

for any $\widetilde{s}>0$ and $(q, r) \in \Lambda_{b}$. Define the operator $\mathcal{G} f:=e^{i t\left(\Delta^{2}+\mu \Delta\right)} f$, then we have

$$
\begin{aligned}
& \mathcal{G}: L^{2} \rightarrow L^{q}\left(\mathbb{R}, L^{r}\right), \\
& \mathcal{G}: H^{4} \rightarrow H^{1, q}\left(\mathbb{R}, L^{r}\right) .
\end{aligned}
$$

Moreover, from the interpolation theorem and Lemma 3.3, we have

$$
\mathcal{G}:\left(L^{2}, H^{4}\right)_{s / 4,2} \rightarrow\left(L^{q}\left(\mathbb{R}, L^{r}\right) \cap H^{1, q}\left(\mathbb{R}, L^{r}\right)\right)_{s / 4,2}=B_{q, 2}^{s / 4}\left(\mathbb{R}, L^{r}\right) .
$$

The inequality (1.4) is now an immediate consequence of (3.2), (3.3) and $\left(L^{2}, H^{4}\right)_{s / 4,2}$ $=H^{s}$.

In what follows, we prove the estimate (1.5). Taking the Fourier transform, we get

$$
(G(f g))^{\wedge}(t, \xi)=\int_{-\infty}^{\infty} \frac{e^{i t \tau}-e^{i t\left(|\xi|^{4}-\mu|\xi|^{2}\right)}}{2 \pi i\left(\tau-|\xi|^{4}+\mu|\xi|^{2}\right)} \widetilde{f g}(\tau, \xi) d \tau
$$

From (3.4) and $\phi_{j} *_{t} e^{i t a}=e^{i t a} \hat{\phi}_{j}(a), \forall a \in \mathbb{R}$, we obtain, for any $j \geq 1$,

$$
\begin{aligned}
& \phi_{j} *_{t}(G(f g))^{\wedge}(t, \xi) \\
= & \int_{-\infty}^{\infty} \frac{e^{i t \tau} \hat{\phi}_{j}(\tau)}{2 \pi i\left(\tau-|\xi|^{4}+\mu|\xi|^{2}\right)} \widetilde{f g}(\tau, \xi) d \tau \\
& -\int_{-\infty}^{\infty} \frac{e^{i t\left(|\xi|^{4}-\mu|\xi|^{2}\right)} \hat{\phi}_{j}\left(|\xi|^{4}-\mu|\xi|^{2}\right) \hat{\chi}_{j}(\tau)}{2 \pi i\left(\tau-|\xi|^{4}+\mu|\xi|^{2}\right)} \widetilde{f g}(\tau, \xi) d \tau \\
& -\int_{-\infty}^{\infty} \frac{e^{i t\left(|\xi|^{4}-\mu|\xi|^{2}\right)} \hat{\phi}_{j}\left(|\xi|^{4}-\mu|\xi|^{2}\right)\left(1-\hat{\chi}_{j}(\tau)\right)}{2 \pi i\left(\tau-|\xi|^{4}+\mu|\xi|^{2}\right)} \cdot \hat{\chi}_{j}\left(|\xi|^{4}-\mu|\xi|^{2}\right) \widetilde{f g}(\tau, \xi) d \tau
\end{aligned}
$$

where we also used the fact that $\hat{\chi}_{k}=1$ on the support of $\hat{\phi}_{k}$. Moreover, since

$$
\mathcal{F}_{\tau}^{-1}\left\{\frac{1}{i\left(\tau-|\xi|^{4}+\mu|\xi|^{2}\right)}\right\}(t)=\frac{1}{2} \operatorname{sign}(t) e^{i t\left(|\xi|^{4}-\mu|\xi|^{2}\right)},
$$

we obtain

$$
\phi_{j} *_{t}(G(f g))
$$




$$
\begin{aligned}
= & \frac{1}{2} \int_{-\infty}^{\infty} \operatorname{sign}(t-\tau) e^{i(t-\tau)\left(\Delta^{2}+\mu \Delta\right)}\left(\phi_{j} *_{t}(f g)\right)(\tau) d \tau \\
& -\frac{1}{2} e^{i t\left(\Delta^{2}+\mu \Delta\right)} \int_{-\infty}^{\infty} \operatorname{sign}(-\tau) e^{i \tau\left(\Delta^{2}+\mu \Delta\right)}\left(\phi_{j / 4} *_{x} \chi_{j} *_{t}(f g)\right)(\tau) d \tau \\
& -\left.e^{i t\left(\Delta^{2}+\mu \Delta\right)}\left\{K_{j} *_{t, x} \chi_{j / 4} *_{x}(f g)\right\}\right|_{t=0},
\end{aligned}
$$

where $K_{j}$ is the function defined in Lemma 3.1

We first prove that

$$
\left(\sum_{j \geq 1}\left(2^{j s / 4}\left\|K_{j} *_{t, x} \chi_{j / 4} *_{x}(f g)\right\|_{L^{\infty} L^{2} \cap L^{q} L^{r}}\right)^{2}\right)^{1 / 2} \lesssim\|f g\|_{L^{\bar{q}} L^{\bar{r}}} .
$$

Let $q_{0}, r_{0}, q_{1}, r_{1}$ be given by the equation $1=\frac{1}{q_{0}}+\frac{1}{\bar{q}}, 1+\frac{1}{2}=\frac{1}{r_{0}}+\frac{1}{\bar{r}}, 1+\frac{1}{q}=\frac{1}{q_{1}}+\frac{1}{\bar{q}}$ and $1+\frac{1}{r}=\frac{1}{r_{1}}+\frac{1}{\bar{r}}$. Then it is easy to check that $1 \leq q_{0}, r_{0}, q_{1}, r_{1} \leq \infty$ and $\frac{4}{q_{0}}-N\left(1-\frac{1}{r_{0}}\right)=$ $\frac{4}{q_{1}}-N\left(1-\frac{1}{r_{1}}\right)=s$. Thus from Young's inequality and Lemma 3.1, we have

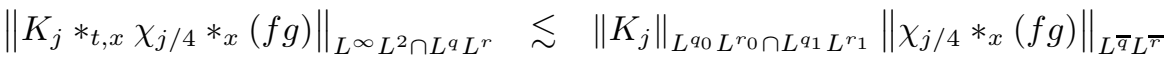

$$
\begin{aligned}
& \lesssim 2^{-j s / 4}\left\|\chi_{j / 4} *_{x}(f g)\right\|_{L^{\bar{q}} L^{\bar{r}}} \text {. }
\end{aligned}
$$

Since $1 \leq \bar{q}, \bar{r} \leq 2$, it follows from (3.7), Minkowski's inequality and Sobolev's embedding $L^{\bar{r}}\left(\mathbb{R}^{N}\right) \hookrightarrow B_{\bar{r}, 2}^{0}\left(\mathbb{R}^{N}\right)$ that

$$
\begin{aligned}
& \left(\sum_{j \geq 1}\left(2^{j s / 4}\left\|K_{j} *_{t, x} \chi_{j / 4} *_{x}(f g)\right\|_{L^{q} L^{r}}\right)^{2}\right)^{1 / 2} \\
\lesssim & \left(\sum_{j \geq 1}\left\|\chi_{j / 4} *_{x}(f g)\right\|_{L^{\bar{q}} L^{\bar{r}}}^{2}\right)^{1 / 2} \lesssim\|f g\|_{L^{\bar{q}} B_{\bar{r}, 2}^{0}} \lesssim\|f g\|_{L^{\bar{q}} L^{\bar{r}}} .
\end{aligned}
$$

Next, we prove that

$$
\begin{aligned}
& \left(\sum_{j \geq 1}\left(2^{j s / 4}\left\|\int_{-\infty}^{\infty} \operatorname{sign}(t-\tau) e^{i(t-\tau)\left(\Delta^{2}+\mu \Delta\right)}\left(\phi_{j} *_{t}(f g)\right) d \tau\right\|_{L^{q} L^{r}}\right)^{2}\right)^{1 / 2} \\
& \lesssim\left(\int_{-\infty}^{\infty}\left(|\tau|^{-s / 4}\|g(t-\tau)(f(t-\tau)-f(t))\|_{L^{\gamma_{0}^{\prime} L^{\rho_{0}^{\prime}}}}\right)^{2} \frac{d \tau}{|\tau|}\right)^{1 / 2} \\
& +\left(\int_{-\infty}^{\infty}\left(|\tau|^{-s / 4}\|(g(t-\tau)-g(t)) f(t)\|_{L^{\gamma_{1}^{\prime}} L^{\rho_{1}^{\prime}}}\right)^{2} \frac{d \tau}{|\tau|}\right)^{1 / 2} .
\end{aligned}
$$

Since $\int_{-\infty}^{\infty} \phi(t) d t=\hat{\phi}(0)=0$, we have

$$
\begin{aligned}
\phi_{j} *_{t}(f g)(t)= & 2^{j} \int_{-\infty}^{\infty} \phi\left(2^{j} \tau\right) g(t-\tau)(f(t-\tau)-f(t)) d \tau \\
& +2^{j} \int_{-\infty}^{\infty} \phi\left(2^{j} \tau\right)(g(t-\tau)-g(t)) f(t) d \tau .
\end{aligned}
$$


From (3.10) and Strichartz's estimate (2.4), we get

$$
\begin{aligned}
& \sum_{j \geq 1}\left(2^{j s / 4}\left\|\int_{-\infty}^{\infty} \operatorname{sign}(t-\tau) e^{i(t-\tau)\left(\Delta^{2}+\mu \Delta\right)}\left(\phi_{j} *_{t}(f g)\right) d \tau\right\|_{L^{q} L^{r}}\right)^{2} \\
\lesssim & \sum_{j \geq 1} 2^{j(s / 2+2)}\left(\int_{-\infty}^{\infty}\left|\phi\left(2^{j} \tau\right)\right|\|g(t-\tau)(f(t-\tau)-f(t))\|_{L^{\gamma_{0}^{\prime}} L^{\rho_{0}^{\prime}}} d \tau\right)^{2} \\
& +\sum_{j \geq 1} 2^{j(s / 2+2)}\left(\int_{-\infty}^{\infty}\left|\phi\left(2^{j} \tau\right)\right|\|(g(t-\tau)-g(t)) f(t)\|_{L^{\gamma_{1}^{\prime}} L^{\rho_{1}^{\prime}}} d \tau\right)^{2} \\
=: & I+I I .
\end{aligned}
$$

For the estimate of $I$, we have, by using Cauchy-Schwartz inequality,

$$
\begin{aligned}
& \left(\int_{-\infty}^{\infty}\left|\phi\left(2^{j} \tau\right)\right|\|g(t-\tau)(f(t-\tau)-f(t))\|_{L^{\gamma_{0}^{\prime} L^{\rho_{0}^{\prime}}}} d \tau\right)^{2} \\
& \lesssim \int_{2^{j}|\tau| \leq 1}\left(\phi\left(2^{j} \tau\right)\right)^{2} d \tau \int_{2^{j}|\tau| \leq 1}\|g(t-\tau)(f(t-\tau)-f(t))\|_{L^{\gamma_{0}^{\prime}} L^{\rho_{0}^{\prime}}}^{2} d \tau \\
& +\int_{2^{j}|\tau| \geq 1}|\tau|^{3}\left(\phi\left(2^{j} \tau\right)\right)^{2} d \tau \int_{2^{j}|\tau| \geq 1} \frac{\|g(t-\tau)(f(t-\tau)-f(t))\|_{L^{\gamma_{0}^{\prime}} L^{\rho_{0}^{\prime}}}^{2}}{|\tau|^{3}} d \tau \\
& \lesssim 2^{-j} \int_{2^{j}|\tau| \leq 1}\|g(t-\tau)(f(t-\tau)-f(t))\|_{L^{\gamma_{0}^{\prime} L^{\rho_{0}^{\prime}}}}^{2} d \tau \\
& +2^{-4 j} \int_{2^{j}|\tau| \geq 1} \frac{\|g(t-\tau)(f(t-\tau)-f(t))\|_{L^{\gamma_{0}^{\prime}} L^{\rho_{0}^{\prime}}}^{2}}{|\tau|^{3}} d \tau .
\end{aligned}
$$

This inequality together with Fubini's Theorem yields

$$
\begin{aligned}
& I \lesssim \sum_{j \geq 1} 2^{j(s / 2+1)} \int_{2^{j}|\tau| \leq 1}\|g(t-\tau)(f(t-\tau)-f(t))\|_{L^{\gamma_{0}^{\prime} L^{\rho_{0}^{\prime}}}}^{2} d \tau \\
& +\sum_{j \geq 1} 2^{j(s / 2-2)} \int_{2^{j}|\tau| \geq 1} \frac{\|g(t-\tau)(f(t-\tau)-f(t))\|_{L^{\gamma_{0}^{\prime}} L^{\rho_{0}^{\prime}}}^{2}}{|\tau|^{3}} d \tau \\
& \lesssim \int_{-\infty}^{\infty} \sum_{2^{j}|\tau| \leq 1} 2^{j(s / 2+1)}\|g(t-\tau)(f(t-\tau)-f(t))\|_{L^{\gamma_{0}^{\prime} L^{\rho_{0}^{\prime}}}}^{2} d \tau \\
& +\int_{-\infty}^{\infty} \sum_{2^{j}|\tau| \geq 1} 2^{j(s / 2-2)} \frac{\|g(t-\tau)(f(t-\tau)-f(t))\|_{L^{\gamma_{0}^{\prime}} L^{\rho_{0}^{\prime}}}^{2}}{|\tau|^{3}} d \tau \\
& \lesssim \int_{-\infty}^{\infty}\left(|\tau|^{-s / 4}\|g(t-\tau)(f(t-\tau)-f(t))\|_{L^{\gamma_{0}^{\prime} L^{\rho_{0}^{\prime}}}}\right)^{2} \frac{d \tau}{|\tau|} .
\end{aligned}
$$

Similarly, we have

$$
I I \lesssim \int_{-\infty}^{\infty}\left(|\tau|^{-s / 4}\|(g(t-\tau)-g(t)) f(t)\|_{L^{\gamma_{1}^{\prime}} L^{\rho_{1}^{\prime}}}\right)^{2} \frac{d \tau}{|\tau|} .
$$

The inequality (3.9) is now an immediate consequence of (3.11), (3.12) and (3.13). 
Using the same method as that used to derive (3.9), we obtain

$$
\begin{aligned}
& \left(\sum_{j \geq 1}\left(2^{j s / 4}\left\|e^{i t\left(\Delta^{2}+\mu \Delta\right)} \int_{-\infty}^{\infty} \operatorname{sign}(-\tau) e^{i \tau\left(\Delta^{2}+\mu \Delta\right)}\left(\phi_{j / 4} *_{x} \chi_{j} *_{t}(f g)\right)(\tau) d \tau\right\|_{L^{q} L^{r}}\right)^{2}\right)^{1 / 2} \\
& \lesssim\left(\int_{-\infty}^{\infty}\left(|\tau|^{-s / 4}\|g(t-\tau)(f(t-\tau)-f(t))\|_{L^{\gamma_{0}^{\prime}} L^{\rho_{0}^{\prime}}}\right)^{2} \frac{d \tau}{|\tau|}\right)^{1 / 2} \\
& \quad+\left(\int_{-\infty}^{\infty}\left(|\tau|^{-s / 4}\|(g(t-\tau)-g(t)) f(t)\|_{L^{\gamma_{1}^{\prime}}}{ }_{L^{\rho_{1}^{\prime}}}\right)^{2} \frac{d \tau}{|\tau|}\right)^{1 / 2}
\end{aligned}
$$

Since $\left\|\psi *_{t} G(f g)\right\|_{L^{q} L^{r}} \lesssim\|f g\|_{L^{\gamma^{\prime}} L^{\rho^{\prime}}}$ by Young's inequality and Strichartz's estimate (2.3), it follows from (3.5), (3.6), (3.9), (3.14) and Strichartz's estimate (2.2) that

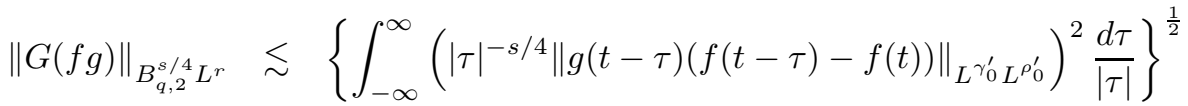

$$
\begin{aligned}
& +\left\{\int_{-\infty}^{\infty}\left(|\tau|^{-s / 4}\|(g(t-\tau)-g(t)) f(t)\|_{L^{\gamma_{1}^{\prime}} L^{\rho_{1}^{\prime}}}\right)^{2} \frac{d \tau}{|\tau|}\right\}^{\frac{1}{2}} \\
& +\|f g\|_{L^{\gamma^{\prime}} L^{\rho^{\prime} \cap L^{\bar{q}} L^{\bar{r}}}} \text {. }
\end{aligned}
$$

Finally, we estimate $\|G(f g)\|_{L^{q} B_{r, 2}^{s}}$. Similar to (3.5), we can write

$$
\begin{aligned}
\phi_{j / 4} *_{x}(G(f g))= & \frac{1}{2} \int_{-\infty}^{\infty} \operatorname{sign}(t-\tau) e^{i(t-\tau)\left(\Delta^{2}+\mu \Delta\right)}\left(\phi_{j / 4} *_{x} \chi_{j} *_{t}(f g)\right)(\tau) d \tau \\
& +K_{j} *_{t, x} \chi_{j / 4} *_{x}(f g) \\
& -\frac{1}{2} e^{i t\left(\Delta^{2}+\mu \Delta\right)} \int_{-\infty}^{\infty} \operatorname{sign}(-\tau) e^{i \tau\left(\Delta^{2}+\mu \Delta\right)}\left(\phi_{j / 4} *_{x} \chi_{j} *_{t}(f g)\right)(\tau) d \tau \\
& -\left.e^{i t\left(\Delta^{2}+\mu \Delta\right)}\left\{K_{j} *_{t, x} \chi_{j / 4} *_{x}(f g)\right\}\right|_{t=0} .
\end{aligned}
$$

Since $\int_{-\infty}^{\infty} \chi(\tau) d \tau=\hat{\chi}(0)=0$, we can apply the equivalent norm in (3.1) and the same argument as that used to derive (3.15) to obtain

$$
\begin{aligned}
\|G(f g)\|_{L^{q} B_{r, 2}^{s}} \lesssim & \left\{\int_{-\infty}^{\infty}\left(|\tau|^{-s / 4}\|g(t-\tau)(f(t-\tau)-f(t))\|_{L^{\gamma_{0}^{\prime} L^{\rho_{0}^{\prime}}}}\right)^{2} \frac{d \tau}{|\tau|}\right\}^{\frac{1}{2}} \\
& +\left\{\int_{-\infty}^{\infty}\left(|\tau|^{-s / 4}\|(g(t-\tau)-g(t)) f(t)\|_{L^{\gamma_{1}^{\prime}} L^{\rho_{1}^{\prime}}}\right)^{2} \frac{d \tau}{|\tau|}\right\}^{\frac{1}{2}} \\
& +\|f g\|_{L^{\gamma^{\prime}} L^{\rho^{\prime} \cap L^{\bar{q}} L^{\bar{r}}} .}
\end{aligned}
$$

This inequality together with (3.15) finishes the proof of Proposition 1.1.

\section{Nonlinear estimates}

In this section, we prove the following lemmas, which provides an estimate for the nonlinearity in the Strichartz spaces. Before stating the Lemmas, we define

$$
1_{\alpha<1}=\left\{\begin{array}{ll}
1, & \text { if } 0<\alpha<1 ; \\
0, & \text { if } \alpha \geq 1,
\end{array} \quad 4^{*}= \begin{cases}\frac{2 N}{N-4}, & \text { if } N \geq 5, \\
\infty, & \text { if } 1 \leq N \leq 4 .\end{cases}\right.
$$


We also define the norm,

$$
\begin{aligned}
\|u\|_{\mathcal{X}^{s}} & :=\sup _{(q, r) \in \Lambda_{b}}\|u\|_{L^{q} B_{r, 2}^{s} \cap B_{q, 2}^{s / 4} L^{r}}, \\
\|u\|_{L_{\mathrm{uloc}, T}^{q} L^{p}} & :=\sup _{b-a=2 T}\left(\int_{a}^{b}\|u\|_{L^{p}\left(\mathbb{R}^{N}\right)}^{q} \mathrm{~d} t\right)^{1 / q},
\end{aligned}
$$

where $s>0,1 \leq p, q<\infty$ and $T>0$. In the rest of this paper, we fix the cut off function $\chi \in C_{0}^{\infty}((-2,2))$ with $\left.\chi\right|_{t \in[-1,1]}=1, \chi_{T}(t)=\chi\left(\frac{t}{T}\right)$. We first consider the case $s<\frac{N}{2}$.

Lemma 4.1. Let $N \geq 1, \beta>\max \left\{2, \frac{N}{4}\right\}, 0<s \leq 2, s<\frac{N}{2}, 0<\alpha,(N-2 s) \alpha<8-\frac{2 N}{\beta}$, $f \in \mathcal{C}(\alpha)$, and $K(x) \in L^{1}\left(\mathbb{R}^{N}\right) \cap L^{\beta}\left(\mathbb{R}^{N}\right)$. There exist $(\gamma, \rho) \in \Lambda_{b}$ and $\sigma>0$ such that

$$
\begin{aligned}
& \left(\int_{-\infty}^{\infty}\left(|\tau|^{-s / 4}\left\|\left(\chi_{T}(t-\tau)-\chi_{T}(\tau)\right) K(x)(f(u)-f(v))\right\|_{L^{\gamma^{\prime} L^{\rho^{\prime}}}}\right)^{2} \frac{\mathrm{d} \tau}{|\tau|}\right)^{1 / 2} \\
\lesssim & T^{\sigma}\left(\|u\|_{\mathcal{X}^{s}}^{\alpha}+\|v\|_{\mathcal{X}^{s}}^{\alpha}\right)\|u-v\|_{\mathcal{X}^{s}} .
\end{aligned}
$$

Proof. Let $b=\frac{N}{\beta}$, then we have $0<b<\min \left\{\frac{N}{2}, 4\right\}$ and $(N-2 s) \alpha<8-2 b$. We first claim that if $(\gamma, \rho),(q, r) \in \Lambda_{b}$ are two admissible pairs that satisfy

$$
\left\{\begin{array}{l}
1-\frac{1}{\gamma}-\frac{\alpha+1}{q}-\frac{s}{4}>0 \\
r<\frac{N}{s} \\
1-\frac{1}{\rho}-(\alpha+1)\left(\frac{1}{r}-\frac{s}{N}\right)>\frac{b}{N}
\end{array}\right.
$$

then the inequality (4.1) holds with $\sigma=1-\frac{1}{\gamma}-\frac{\alpha+1}{q}-\frac{s}{4}$. In fact, let $p, l$ be given by $1-\frac{1}{\rho}=\frac{1}{p}+(\alpha+1)\left(\frac{1}{r}-\frac{s}{N}\right)$ and $1-\frac{1}{\gamma}=\frac{\alpha+1}{q}+\frac{1}{l}$, respectively. Then it is easy to check that $1<l<\infty$ and $1<p<\frac{N}{b}=\beta$; so that $K(x) \in L^{1}\left(\mathbb{R}^{N}\right) \cap L^{\beta}\left(\mathbb{R}^{N}\right) \subset L^{p}\left(\mathbb{R}^{N}\right)$. From (1.7), Hölder's inequality and Sobolev's embedding $B_{r, 2}^{s}\left(\mathbb{R}^{N}\right) \hookrightarrow L^{\frac{N r}{N-s r}}\left(\mathbb{R}^{N}\right)$, we have

$$
\begin{aligned}
& \left\|\left(\chi_{T}(t-\tau)-\chi_{T}(t)\right) K(f(u)-f(v))\right\|_{L^{\gamma^{\prime} L^{\rho^{\prime}}}} \\
\lesssim & \left\|\chi_{T}(t-\tau)-\chi_{T}(t)\right\|_{L^{l}}\|K\|_{L^{p}}\left(\|u\|_{L^{q} L^{\frac{N r}{N-s r}}}^{\alpha}+\|v\|_{L^{q} L^{\frac{N r}{N-s r}}}^{\alpha-s}\right)\|u-v\|_{L^{q} L^{\frac{N r}{N-s r}}} \\
\lesssim & \left\|\chi_{T}(t-\tau)-\chi_{T}(t)\right\|_{L^{l}}\left(\|u\|_{L^{q} B_{r, 2}^{s}}^{\alpha}+\|v\|_{L^{q} B_{r, 2}^{s}}^{\alpha}\right)\|u-v\|_{L^{q} B_{r, 2}^{s}} .
\end{aligned}
$$

Moreover, from (4.5), we have

$$
\begin{aligned}
& \left(\int_{-\infty}^{\infty}\left(|\tau|^{-s / 4}\left\|\left(\chi_{T}(t-\tau)-\chi_{T}(\tau)\right) K(x)(f(u)-f(v))\right\|_{L^{\gamma^{\prime}} L^{\rho^{\prime}}}\right)^{2} \frac{\mathrm{d} \tau}{|\tau|}\right)^{1 / 2} \\
\lesssim & \left\|\chi_{T}\right\|_{B_{l, 2}^{s / 4}}\left(\|u\|_{L^{q} B_{r, 2}^{s}}^{\alpha}+\|v\|_{L^{q} B_{r, 2}^{s}}^{\alpha}\right)\|u-v\|_{L^{q} B_{r, 2}^{s}} .
\end{aligned}
$$

The inequality (4.1) is now an immediate consequence of (4.6) and $\left\|\chi_{T}\right\|_{B_{l, 2}^{s / 4}} \lesssim C_{\chi} T^{\frac{1}{T^{-\frac{s}{4}}}}=$ $C_{\chi} T^{1-\frac{1}{\gamma}-\frac{\alpha+1}{q}-\frac{s}{4}}$.

To prove Lemma 4.1 it suffices to provide two biharmonic admissible pairs $(\gamma, \rho),(q, r) \in$ $\Lambda_{b}$ that satisfy (4.2)-(4.4). We consider four cases. 
Cases 1: $(N-2 s) \alpha<2 s-2 b$. Let $\gamma=q=\infty, \rho=r=2$. Then it is easy to check that $(\gamma, \rho),(q, r) \in \Lambda_{b}$ and (4.2), (4.3) hold. For (4.4), we have

$$
1-\frac{1}{\rho}-(\alpha+1)\left(\frac{1}{r}-\frac{s}{N}\right)-\frac{b}{N}=\frac{2 s-2 b-(N-2 s) \alpha}{2 N}>0 .
$$

Cases 2: $2 s-2 b \leq(N-2 s) \alpha<4+2 s-2 b$. Let $q=\infty, r=2$ and

$$
\gamma=\frac{8}{(N-2 s) \alpha-2 s+2 b+\varepsilon}, \quad \rho=\frac{2 N}{N+2 s-2 b-(N-2 s) \alpha-\varepsilon},
$$

where $\varepsilon>0$ sufficiently small such that

$$
\varepsilon<\min \{4+2 s-2 b-(N-2 s) \alpha, 8-2 b-(N-2 s) \alpha, 2\} .
$$

Then it is easy to check that $(\gamma, \rho),(q, r) \in \Lambda_{b}$ and (4.3) hold. Moreover, by direct computation, we have

$$
\left\{\begin{array}{l}
1-\frac{1}{\gamma}-\frac{\alpha+1}{q}-\frac{s}{4}=\frac{8-2 b-(N-2 s) \alpha-\varepsilon}{8}>0, \\
1-\frac{1}{\rho}-(\alpha+1)\left(\frac{1}{r}-\frac{s}{N}\right)=\frac{2 b+\varepsilon}{2 N}>\frac{b}{N} .
\end{array}\right.
$$

Hence we see that (4.2) and (4.4) hold.

Cases 3: $(N-2 s) \alpha \geq 4+2 s-2 b$ and $N \geq 5$. Let $\gamma=2, \rho=\frac{2 N}{N-4}$, and

$$
q=\frac{8(\alpha+1)}{(N-2 s) \alpha-(4+2 s-2 b)+2 \varepsilon}, \quad r=\frac{2 N(\alpha+1)}{N+2 s(\alpha+1)+4-2 b-2 \varepsilon},
$$

where $\varepsilon>0$ sufficiently small such that

$$
\varepsilon<\min \left\{\frac{8-2 b-(N-2 s) \alpha}{2}, s\right\} .
$$

Then it is easy to check that and Then by direct calculation, we have $(\gamma, \rho),(q, r) \in \Lambda_{b}$ and

$$
\left\{\begin{array}{l}
1-\frac{1}{\gamma}-\frac{\alpha+1}{q}-\frac{s}{4}=\frac{8-2 b-(N-2 s) \alpha-2 \varepsilon}{N(N+4-2 b-2 \varepsilon)}>0, \\
\frac{N}{s}-r=\frac{N}{s(N+2 s(\alpha+1)+4-2 b-2 \varepsilon)}>0, \\
1-\frac{1}{\rho}-(\alpha+1)\left(\frac{1}{r}-\frac{s}{N}\right)=\frac{b+\varepsilon}{N}>\frac{b}{N} .
\end{array}\right.
$$

Hence we see that (4.2)-(4.4) hold.

Cases 4: $(N-2 s) \alpha \geq 4+2 s-2 b$ and $N \leq 4$. Let $\gamma=\frac{8}{N(1-\varepsilon)}, \rho=\frac{2}{\varepsilon}$, and

$$
q=\frac{8(\alpha+1)}{(N-2 s) \alpha-(1-2 \varepsilon) N-2 s+2 b}, \quad r=\frac{N(\alpha+1)}{(1-\varepsilon) N-b+s(\alpha+1)},
$$

where $\varepsilon>0$ sufficiently small such that

$$
\varepsilon<\min \left\{\frac{N-b}{N}, \frac{8-2 b-(N-2 s) \alpha}{N}\right\} .
$$

Then by direct calculation, we have $(\gamma, \rho),(q, r) \in \Lambda_{b}$ and

$$
\left\{\begin{array}{l}
1-\frac{1}{\gamma}-\frac{\alpha+1}{q}-\frac{s}{4}=\frac{8-2 b-\varepsilon N-(N-2 s) \alpha}{8}>0, \\
\frac{N}{s}-r=\frac{N(1-\varepsilon) N-b)}{s((1-\varepsilon) N-b+s(\alpha+1))}>0 \\
1-\frac{1}{\rho}-(\alpha+1)\left(\frac{1}{r}-\frac{s}{N}\right)=\frac{2 b+N \varepsilon}{2 N}>\frac{b}{N} .
\end{array}\right.
$$

Hence we see that (4.2)-(4.4) hold. 
Lemma 4.2. Let $N \geq 1, \beta>\max \left\{2, \frac{N}{4}\right\}, 0<s \leq 2, s<\frac{N}{2}, 0<\alpha,(N-2 s) \alpha<8-\frac{2 N}{\beta}$, $f \in \mathcal{C}(\alpha)$, and $K(x) \in L^{\infty}\left(\mathbb{R}^{N}\right)$. There exist $(\gamma, \rho) \in \Lambda_{b}$ and $\sigma>0$ such that

$$
\begin{aligned}
& \left(\int_{-\infty}^{\infty}\left(|\tau|^{-s / 4}\left\|\left(\chi_{T}(t-\tau)-\chi_{T}(\tau)\right) K(x)(f(u)-f(v))\right\|_{L^{\gamma^{\prime} L^{\rho^{\prime}}}}\right)^{2} \frac{\mathrm{d} \tau}{|\tau|}\right)^{1 / 2} \\
\lesssim & T^{\sigma}\left(\|u\|_{\mathcal{X}^{s}}^{\alpha}+\|v\|_{\mathcal{X}^{s}}^{\alpha}\right)\|u-v\|_{\mathcal{X}^{s}} .
\end{aligned}
$$

Proof. We first claim that if $(\gamma, \rho),(q, r) \in \Lambda_{b}$ are two admissible pairs that satisfy

$$
\left\{\begin{array}{l}
1-\frac{1}{\gamma}-\frac{\alpha+1}{q}-\frac{s}{4}>0, \\
r<\frac{N}{s} \\
\frac{\alpha+1}{r}>1-\frac{1}{\rho}>(\alpha+1)\left(\frac{1}{r}-\frac{s}{N}\right),
\end{array}\right.
$$

then the inequality (4.7) holds with $\sigma=1-\frac{1}{\gamma}-\frac{\alpha+1}{q}-\frac{s}{4}$. In fact, let $p, l$ be given by $1-\frac{1}{\rho}=\frac{\alpha+1}{p}$ and $1-\frac{1}{\gamma}=\frac{\alpha+1}{q}+\frac{1}{l}$, respectively. Then by (4.10) we have $\frac{1}{r}-\frac{s}{N}<\frac{1}{p}<\frac{1}{r}$; so that the embedding $B_{r, 2}^{s}\left(\mathbb{R}^{N}\right) \hookrightarrow L^{p}\left(\mathbb{R}^{N}\right)$ holds. Similar to (4.6), we deduce from Hölder's inequality and Sobolev's embedding $B_{r, 2}^{s}\left(\mathbb{R}^{N}\right) \hookrightarrow L^{p}\left(\mathbb{R}^{N}\right)$ that

$$
\begin{aligned}
& \left(\int_{-\infty}^{\infty}\left(|\tau|^{-s / 4}\left\|\left(\chi_{T}(t-\tau)-\chi_{T}(\tau)\right) K(x)(f(u)-f(v))\right\|_{L^{\gamma^{\prime} L^{\rho^{\prime}}}}\right)^{2} \frac{\mathrm{d} \tau}{|\tau|}\right)^{1 / 2} \\
\lesssim & \left\|\chi_{T}\right\|_{B_{l, 2}^{s / 4}}\left(\|u\|_{L^{q} B_{r, 2}^{s}}^{\alpha}+\|v\|_{L^{q} B_{r, 2}^{s}}^{\alpha}\right)\|u-v\|_{L^{q} B_{r, 2}^{s}},
\end{aligned}
$$

where we also used the boundedness of $K(x)$. The inequality (4.7) is now an immediate consequence of (4.11) and $\left\|\chi_{T}\right\|_{B_{l, 2}^{s / 4}} \lesssim C_{\chi} T^{\frac{1}{l}-\frac{s}{4}}=C_{\chi} T^{1-\frac{1}{\gamma}-\frac{\alpha+1}{q}-\frac{s}{4}}$.

To prove Lemma 4.2 it suffices to provide two biharmonic admissible pairs $(\gamma, \rho),(q, r) \in$ $\Lambda_{b}$ that satisfy (4.8)-(4.10). We consider four cases.

Cases 1: $(N-2 s) \alpha<2 s$. Let $\gamma=q=\infty, \rho=r=2$.

Cases 2: $2 s \leq(N-2 s) \alpha<4+2 s$. Let $q=\infty, r=2$ and

$$
\gamma=\frac{8}{(N-2 s) \alpha-2 s+\varepsilon}, \quad \rho=\frac{2 N}{N+2 s-(N-2 s) \alpha-\varepsilon},
$$

where $\varepsilon>0$ sufficiently small such that

$$
\varepsilon<\min \left\{4+2 s-(N-2 s) \alpha, 8-(N-2 s) \alpha, \frac{2 s(\alpha+1)}{N}\right\} .
$$

Cases 3: $(N-2 s) \alpha \geq 4+2 s$ and $N \geq 5$. Let $\gamma=2, \rho=\frac{2 N}{N-4}$, and

$$
q=\frac{8(\alpha+1)}{(N-2 s) \alpha-(4+2 s)+2 \varepsilon}, \quad r=\frac{2 N(\alpha+1)}{N+2 s(\alpha+1)+4-2 \varepsilon},
$$

where $\varepsilon>0$ sufficiently small such that

$$
\varepsilon<\min \left\{\frac{8-(N-2 s) \alpha}{2}, s\right\} .
$$


Cases 4: $(N-2 s) \alpha \geq 4+2 s$ and $N \leq 4$. Let $\gamma=\frac{8}{N(1-\varepsilon)}, \rho=\frac{2}{\varepsilon}$, and

$$
q=\frac{8(\alpha+1)}{(N-2 s) \alpha-(1-2 \varepsilon) N-2 s}, \quad r=\frac{N(\alpha+1)}{(1-\varepsilon) N+s(\alpha+1)},
$$

where $\varepsilon>0$ sufficiently small such that

$$
\varepsilon<\min \left\{\frac{1}{2}, \frac{8-(N-2 s) \alpha}{N}\right\} .
$$

In each case, we can verify that $(\gamma, \rho),(q, r) \in \Lambda_{b}$, (4.8)-(4.10) hold and omit the details.

Lemma 4.3. Let $N \geq 1, \beta>\max \left\{2, \frac{N}{4}\right\}, 0<s \leq 2, s<\frac{N}{2}, 0<\alpha,(N-2 s) \alpha<8-\frac{2 N}{\beta}$, $f \in \mathcal{C}(\alpha)$, and $K(x) \in L^{1}\left(\mathbb{R}^{N}\right) \cap L^{\beta}\left(\mathbb{R}^{N}\right)$. There exist $\sigma>0$ and $\widetilde{q}>1,(\gamma, \rho),(q, r) \in \Lambda_{b}$ with $\widetilde{q}<q, r<\min \left\{\frac{N}{s}, 4^{*}\right\}$ such that

$$
\begin{aligned}
& \left(\int_{-\infty}^{\infty}\left(|\tau|^{-s / 4}\left\|\chi_{T}(t-\tau) K(x)\left((f(u)-f(v))_{\tau}-(f(u)-f(v))\right)\right\|_{L^{\gamma^{\prime} L^{\rho^{\prime}}}}\right)^{2} \frac{\mathrm{d} \tau}{|\tau|}\right)^{1 / 2} \\
& \lesssim T^{\sigma}\left(\|u\|_{\mathcal{X}^{s}}^{\alpha}+\|v\|_{\mathcal{X}^{s}}^{\alpha}\right)\|u-v\|_{\mathcal{X}^{s}}+1_{\alpha<1}\|u-v\|_{L_{u l o c, T}^{\tilde{q}} L \frac{N r}{N-s r}}^{\alpha}\|v\|_{\mathcal{X}^{s}}
\end{aligned}
$$

Proof. Let $b=\frac{N}{\beta}$, then we have $0<b<\min \left\{\frac{N}{2}, 4\right\}$ and $(N-2 s) \alpha<8-2 b$. We first claim that if $(\gamma, \rho),(q, r),(m, n) \in \Lambda_{b}$ are three admissible pairs that satisfy

$$
\left\{\begin{array}{l}
1-\frac{1}{\gamma}-\frac{\alpha}{q}-\frac{1}{m}>0 \\
r<\frac{N}{s} \\
1-\frac{1}{\rho}-\alpha\left(\frac{1}{r}-\frac{s}{N}\right)-\frac{1}{n}>\frac{b}{N}
\end{array}\right.
$$

Then we can find $1<\widetilde{q}<q$ such that the inequality (4.12) holds with $\sigma=1-\frac{1}{\gamma}-\frac{\alpha}{q}-\frac{1}{m}$. In fact, from

$$
\begin{aligned}
& \left(f\left(u_{\tau}\right)-f\left(v_{\tau}\right)\right)-(f(u)-f(v)) \\
= & \left(\left(u_{\tau}-v_{\tau}\right)-(u-v)\right) \int_{0}^{1} f^{\prime}\left(u+\theta\left(u_{\tau}-u\right)\right) d \theta \\
& +\left(v_{\tau}-v\right) \int_{0}^{1}\left[f^{\prime}\left(u+\theta\left(u_{\tau}-u\right)\right)-f^{\prime}\left(v+\theta\left(v_{\tau}-v\right)\right)\right] d \theta \\
= & A_{1}+A_{2},
\end{aligned}
$$

we have, by applying (1.7)

$$
\left|A_{1}\right| \lesssim\left|\left(u_{\tau}-v_{\tau}\right)-(u-v)\right|\left(|u|^{\alpha}+\left|u_{\tau}\right|^{\alpha}\right),
$$

and

$$
\left|A_{2}\right| \leq \begin{cases}\left|v_{\tau}-v\right|\left(|u|+\left|u_{\tau}\right|+|v|+\left|v_{\tau}\right|\right)^{\alpha-1}\left(|u-v|+\left|u_{\tau}-v_{\tau}\right|\right), & \text { if } \alpha \geqslant 1 \\ \left|v_{\tau}-v\right|\left(|u-v|^{\alpha}+\left|u_{\tau}-v_{\tau}\right|^{\alpha}\right), & \text { if } 0<\alpha<1 .\end{cases}
$$


Put $1-\frac{1}{\rho}=\frac{1}{p}+\alpha\left(\frac{1}{r}-\frac{s}{N}\right)+\frac{1}{n}$. Then by (4.15), we have $1<p<\frac{N}{b}=\beta$ and thus $K(x) \in L^{1}\left(\mathbb{R}^{N}\right) \cap L^{\beta}\left(\mathbb{R}^{N}\right) \subset L^{p}\left(\mathbb{R}^{N}\right)$. From (4.16), (4.17) and (4.18), the boundedness of $\chi_{T}$ and Hölder's inequality, we get

$$
\begin{aligned}
& \left\|\chi_{T}(t-\tau) K(x)\left((f(u)-f(v))_{\tau}-(f(u)-f(v))\right)\right\|_{L^{\gamma^{\prime}} L^{\rho^{\prime}}} \\
& \lesssim T^{\sigma}\left\|(u-v)_{\tau}-(u-v)\right\|_{L^{m} L^{n}}\|K(x)\|_{L^{p}}\left(\|u\|_{L^{q} L^{\frac{N r}{N-s r}}}^{\alpha}+\|v\|_{L^{q} L^{\frac{N r}{N-s r}}}^{\alpha}\right) \\
& +\left\{\begin{array}{l}
T^{\sigma}\|K(x)\|_{L^{p}}\left\|v_{\tau}-v\right\|_{L^{m} L^{n}}\left(\|u\|_{L^{q} L^{\frac{N r}{N-s r}}}^{\alpha-1}+\|v\|_{L^{q} L^{\frac{N r}{N-s r}}}^{\alpha-1}\right)\|u-v\|_{L^{q} L^{\frac{N r}{N-s r}}}, \alpha \geq 1, \\
\left\|v_{\tau}-v\right\|_{L^{m} L^{n}}\|K(x)\|_{L^{p}}\|u-v\|_{L_{\text {uloc }, T}^{\widetilde{q}} L^{\frac{N r}{N-s r}}}^{\alpha}, 0<\alpha<1,
\end{array}\right.
\end{aligned}
$$

where $\sigma=1-\frac{1}{\gamma}-\frac{\alpha}{q}-\frac{1}{m}$ and $1<\widetilde{q}<q$ is given by $1-\frac{1}{\gamma}=\frac{\alpha}{\tilde{q}}+\frac{1}{m}$. This inequality together with Sobolev's embedding $B_{r, 2}^{s}\left(\mathbb{R}^{N}\right) \hookrightarrow L^{\frac{N r}{N-s r}}\left(\mathbb{R}^{N}\right)$ implies

$$
\begin{aligned}
& \left(\int_{-\infty}^{\infty}\left(|\tau|^{-s / 4}\left\|\chi_{T}(t-\tau) K(x)\left((f(u)-f(v))_{\tau}-(f(u)-f(v))\right)\right\|_{L^{\gamma^{\prime} L^{\rho^{\prime}}}}\right)^{2} \frac{\mathrm{d} \tau}{|\tau|}\right)^{1 / 2} \\
& \lesssim T^{\sigma}\|u-v\|_{B_{m, 2}^{s / 4} L^{n}}\left(\|u\|_{L^{q} B_{r, 2}^{s}}^{\alpha}+\|v\|_{L^{q} B_{r, 2}^{s}}^{\alpha}\right) \\
& \quad+\left\{\begin{array}{l}
T^{\sigma}\|v\|_{B_{m, 2}^{s / 4} L^{n}}\left(\|u\|_{L^{q} B_{r, 2}^{s}}^{\alpha-1}+\|v\|_{L^{q} B_{r, 2}^{s}}^{\alpha-1}\right)\|u-v\|_{L^{q} B_{r, 2}^{s}}, \alpha \geq 1 \\
\|v\|_{B_{m, 2}^{s / 4} L^{n}}\|u-v\|_{L_{\mathrm{uloc}, T}^{\tilde{q}} \frac{N r}{N-s r}}^{\alpha}, 0<\alpha<1 .
\end{array}\right.
\end{aligned}
$$

The inequality (4.12) is now an immediate consequence of (4.19) and Young's inequality.

To prove Lemma 4.3, it suffices to provide three biharmonic admissible pairs $(\gamma, \rho)$, $(q, r),(m, n) \in \Lambda_{b}$ with $r<4^{*}$ that satisfy (4.13)-(4.15). We consider two cases.

Case 1: $N \geq 2 s+4$. Let

$$
\begin{cases}\gamma=2, & \rho=\frac{2 N}{N-4}, \\ q=m=\frac{4(\alpha+1)}{2-\varepsilon}, & r=n=\frac{2 N(\alpha+1)}{N(\alpha+1)-4+2 \varepsilon},\end{cases}
$$

where $\varepsilon>0$ sufficiently small such that

$$
\varepsilon<\min \left\{\frac{8-2 b-(N-2 s) \alpha}{2}, 2\right\} .
$$

Then by direct calculation, we have $(\gamma, \rho),(q, r) \in \Lambda_{b}$ with $r<4^{*}$, and

$$
\left\{\begin{array}{l}
1-\frac{1}{\gamma}-\frac{\alpha}{q}-\frac{1}{m}=\frac{\varepsilon}{4}>0, \\
\frac{N}{s}-r=\frac{N((N-2 s) \alpha+N-2 s-4+2 \varepsilon)}{s(N(\alpha+1)-4+2 \varepsilon)}>0, \\
1-\frac{1}{\rho}-\alpha\left(\frac{1}{r}-\frac{s}{N}\right)-\frac{1}{n}-\frac{b}{N}=\frac{8-2 b-(N-2 s) \alpha-2 \varepsilon}{2 N}>0 .
\end{array}\right.
$$

Hence we see that (4.13)-4.15) hold.

Cases 2: $N<2 s+4$. Let

$$
\begin{cases}\gamma=m=\frac{8}{b+2 \alpha \varepsilon}, & \rho=n=\frac{2 N}{N-b-2 \alpha \varepsilon}, \\ q=\frac{8}{N-2 s-2 \varepsilon}, & r=\frac{N}{s+\varepsilon},\end{cases}
$$

where $\varepsilon>0$ sufficiently small such that

$$
\varepsilon<\min \left\{\frac{N-b}{2 \alpha}, \frac{8-2 b-(N-2 s) \alpha}{2 \alpha}, \frac{N-2 s}{2}\right\} .
$$


Then it is easy to check that $(q, r),(\gamma, \rho) \in \Lambda_{b}$ with $r<\frac{N}{s}<4^{*}$. Moreover, by direct computation, we have

$$
\left\{\begin{array}{l}
1-\frac{1}{\gamma}-\frac{\alpha}{q}-\frac{1}{m}=\frac{8-2 b-(N-2 s) \alpha-2 \varepsilon \alpha}{8}>0, \\
1-\frac{1}{\rho}-\alpha\left(\frac{1}{r}-\frac{s}{N}\right)-\frac{1}{n}-\frac{b}{N}=\frac{\alpha \varepsilon}{N}>0 .
\end{array}\right.
$$

Hence we see that (4.13)-(4.15) hold.

Lemma 4.4. Let $N \geq 1, \beta>\max \left\{2, \frac{N}{4}\right\}, 0<s \leq 2, s<\frac{N}{2}, 0<\alpha,(N-2 s) \alpha<8-\frac{2 N}{\beta}$, $f \in \mathcal{C}(\alpha)$, and $K(x) \in L^{\infty}\left(\mathbb{R}^{N}\right)$. There exist $\sigma>0$ and $\widetilde{q}>1,(\gamma, \rho),(q, r) \in \Lambda_{b}$ with $\widetilde{q}<q, r<\min \left\{\frac{N}{s}, 4^{*}\right\}$ such that

$$
\begin{aligned}
& \left(\int_{-\infty}^{\infty}\left(|\tau|^{-s / 4}\left\|\chi_{T}(t-\tau) K(x)\left((f(u)-f(v))_{\tau}-(f(u)-f(v))\right)\right\|_{L^{\gamma^{\prime}} L^{\rho^{\prime}}}\right)^{2} \frac{\mathrm{d} \tau}{|\tau|}\right)^{1 / 2} \\
& \lesssim T^{\sigma}\left(\|u\|_{\mathcal{X}^{s}}^{\alpha}+\|v\|_{\mathcal{X}^{s}}^{\alpha}\right)\|u-v\|_{\mathcal{X}^{s}}+1_{\alpha<1}\|u-v\|_{L_{u l o c, T}^{\tilde{q}} L^{\frac{N r}{N-s r}}}^{\alpha}\|v\|_{\mathcal{X}^{s}} .
\end{aligned}
$$

Proof. Let

$$
q=\frac{8(\alpha+2)}{(N-2 s) \alpha+2 \varepsilon}, \quad r=\frac{N(\alpha+2)}{N+\alpha s-\varepsilon}
$$

where $\varepsilon>0$ sufficiently small such that

$$
\varepsilon<\min \left\{N-2 s, \frac{8-(N-2 s) \alpha}{2}, \alpha s\right\} .
$$

Then by direct calculation, we have $r<4^{*}$ and

$$
\left\{\begin{array}{l}
1-\frac{\alpha+2}{q}=\frac{8-(N-2 s) \alpha-2 \varepsilon}{8}>0 \\
\frac{N}{s}-r=\frac{N(N-2 s-\varepsilon)}{s(N+\alpha s-\varepsilon)}>0 .
\end{array}\right.
$$

Let $p, \sigma$ be given by $1=\frac{2}{r}+\frac{\alpha}{p}$ and $1=\frac{1}{\sigma}+\frac{\alpha+2}{q}$, respectively. Since

$$
\left\{\begin{array}{l}
1-\frac{2}{r}-\frac{\alpha}{r}=-\frac{\alpha s-\varepsilon}{N}<0, \\
1-\frac{2}{r}-\alpha\left(\frac{1}{r}-\frac{s}{N}\right)=\frac{\varepsilon}{N}>0,
\end{array}\right.
$$

we have $\frac{1}{r}-\frac{s}{N}<\frac{1}{p}<\frac{1}{r}$; so that the embedding $B_{r, 2}^{s}\left(\mathbb{R}^{N}\right) \hookrightarrow L^{p}\left(\mathbb{R}^{N}\right)$ holds. Similar to (4.19), we deduce from Hölder's inequality and Sobolev's embedding $B_{r, 2}^{s}\left(\mathbb{R}^{N}\right) \hookrightarrow L^{p}\left(\mathbb{R}^{N}\right)$ that

$$
\begin{aligned}
& \left(\int_{-\infty}^{\infty}\left(|\tau|^{-s / 4}\left\|\chi_{T}(t-\tau) K(x)\left((f(u)-f(v))_{\tau}-(f(u)-f(v))\right)\right\|_{L^{q^{\prime} L^{r^{\prime}}}}\right)^{2} \frac{\mathrm{d} \tau}{|\tau|}\right)^{1 / 2} \\
& \lesssim T^{\sigma}\|u-v\|_{B_{q, 2}^{s / 4} L^{r}}\left(\|u\|_{L^{q} B_{r, 2}^{s}}^{\alpha}+\|v\|_{L^{q} B_{r, 2}^{s}}^{\alpha}\right) \\
& \quad+ \begin{cases}T^{\sigma}\|v\|_{B_{q, 2}^{s / 4} L^{r}}\left(\|u\|_{L^{q} B_{r, 2}^{s}}^{\alpha-1}+\|v\|_{L^{q} B_{r, 2}^{s}}^{\alpha}\right)\|u-v\|_{L^{q} B_{r, 2}^{s}}, & \alpha \geq 1, \\
\|v\|_{B_{q, 2}^{s / 4} L^{r}}\|u-v\|_{L_{\mathrm{uloc}, T}^{\tilde{q}} L^{\frac{N r}{N-s r}}}^{\alpha-s} & 0<\alpha<1,\end{cases}
\end{aligned}
$$

where $1<\widetilde{q}<q$ is given by $1=\frac{2}{q}+\frac{\alpha}{\widetilde{q}}$. The inequality (4.20) is now an immediate consequence of (4.22) and Young's inequality. 
Lemma 4.5. Let $N \geq 1, \beta>\max \left\{2, \frac{N}{4}\right\}, 0<s \leq 2, s<\frac{N}{2}, 0<\alpha,(N-2 s) \alpha<8-\frac{2 N}{\beta}$, $f \in \mathcal{C}(\alpha)$, and $K(x) \in L^{1}\left(\mathbb{R}^{N}\right) \cap L^{\beta}\left(\mathbb{R}^{N}\right)$. There exists $\sigma>0$ and $1 \leq \bar{q}, \bar{r} \leq 2$ with $\frac{4}{\bar{q}}-N\left(\frac{1}{2}-\frac{1}{\bar{r}}\right)=4-$ such that

$$
\left\|K(x) \chi_{T}(t)(f(u)-f(v))\right\|_{L^{\bar{q}} L^{\bar{r}}} \lesssim T^{\theta}\left(\|u\|_{\mathcal{X}^{s}}^{\alpha}+\|v\|_{\mathcal{X}^{s}}^{\alpha}\right)\|u-v\|_{\mathcal{X}^{s}} .
$$

Proof. Let $b=\frac{N}{\beta}$, then we have $0<b<\min \left\{\frac{N}{2}, 4\right\}$ and $(N-2 s) \alpha<8-2 b$. We first claim that if $1 \leq \bar{q}, \bar{r} \leq 2$ with $\frac{4}{\bar{q}}-N\left(\frac{1}{2}-\frac{1}{\bar{r}}\right)=4-s$ and $(q, r) \in \Lambda_{b}$ is an admissible pair that satisfy

$$
\left\{\begin{array}{l}
\frac{1}{\bar{q}}-\frac{\alpha+1}{q}>0, \\
r<\frac{N}{s}, \\
\frac{1}{\bar{r}}-(\alpha+1)\left(\frac{1}{r}-\frac{s}{N}\right)>\frac{b}{N},
\end{array}\right.
$$

then the inequality (4.23) holds with $\sigma=\frac{1}{\bar{q}}-\frac{\alpha+1}{q}$. In fact, let $1<p<\infty$ be given by $\frac{1}{\bar{r}}=\frac{1}{p}+(\alpha+1)\left(\frac{1}{r}-\frac{s}{N}\right)$, then by (4.26), we have $1<p<\frac{N}{b}=\beta$; so that $K(x) \in$ $L^{1}\left(\mathbb{R}^{N}\right) \cap L^{\beta}\left(\mathbb{R}^{N}\right) \subset L^{p}\left(\mathbb{R}^{N}\right)$. Using (1.7), Hölder's inequality and Sobolev's embedding $B_{r, 2}^{s}\left(\mathbb{R}^{N}\right) \hookrightarrow L^{\frac{N r}{N-s r}}\left(\mathbb{R}^{N}\right)$, we have

$$
\begin{aligned}
& \left\|K(x) \chi_{T}(t)(f(u)-f(v))\right\|_{L^{\bar{q}} L^{\bar{r}}} \\
\lesssim & \|\| K\left\|_{L^{p}}\left(\|u\|_{L^{\frac{N r}{N-s r}}}^{\alpha}+\|v\|_{L^{\frac{N r}{N-s r}}}^{\alpha}\right)\right\| u-v\left\|_{L^{\frac{N r}{N-s r}}}\right\|_{L^{\bar{q}}} \\
\lesssim & T^{\frac{1}{q}-\frac{\alpha+1}{q}}\left(\|u\|_{L^{q} B_{r, 2}^{s}}^{\alpha}+\|v\|_{L^{q} B_{r, 2}^{s}}^{\alpha}\right)\|u-v\|_{L^{q} B_{r, 2}^{s}},
\end{aligned}
$$

which yields (4.23).

To prove Lemma 4.5. it sufficies to provide $1 \leq \bar{q}, \bar{r} \leq 2$ with $\frac{4}{\bar{q}}-N\left(\frac{1}{2}-\frac{1}{\bar{r}}\right)=4-s$ and an admissible pair $(q, r) \in \Lambda_{b}$ that satisfy (4.24)-(4.26). We consider two cases.

Case 1: $N+2 s \geq 4$. Let $\bar{q}=2, \bar{r}=\frac{2 N}{N+4-2 s}$, then we have $1 \leq \bar{q}, \bar{r} \leq 2$ and $\frac{4}{\bar{q}}-N\left(\frac{1}{2}-\frac{1}{\bar{r}}\right)=4-s$. Next, we choose the admissible pair $(q, r) \in \Lambda_{b}$ that satisfy (4.24)(4.26). We consider two subcases.

Subcase 1: $(N-2 s) \alpha<4-2 b$. Let

$$
q=\infty, \quad r=2 .
$$

Then it is easy to verify that $(q, r) \in \Lambda_{b}$ and (4.24)-(4.26) hold.

Subcase 2: $(N-2 s) \alpha \geq 4-2 b$. Let

$$
q=\frac{8(\alpha+1)}{(N-2 s) \alpha-4+2 b+2 \varepsilon}, \quad r=\frac{2 N(\alpha+1)}{N+4-2 b-2 \varepsilon+2 s \alpha},
$$

where $\varepsilon>0$ sufficiently small such that

$$
\varepsilon<\min \left\{\frac{8-2 b-(N-2 s) \alpha}{2}, 2 \alpha, \frac{N+4-2 b-2 s}{2}\right\} .
$$

Then we have $(q, r) \in \Lambda_{b}$ and

$$
\left\{\begin{array}{l}
\frac{1}{\bar{q}}-\frac{\alpha+1}{q}=\frac{8-2 b-2 \varepsilon-(N-2 s) \alpha}{8}>0 \\
\frac{N}{s}-r=\frac{N(N+4-2 b-2 s-2 \varepsilon)}{s(N+4-2 b-2 \varepsilon+2 s \alpha)}>0 \\
\frac{1}{\bar{r}}-(\alpha+1)\left(\frac{1}{r}-\frac{s}{N}\right)=\frac{b+\varepsilon}{N}>\frac{b}{N} .
\end{array}\right.
$$


Hence we see that (4.24)-(4.26) hold.

Case 2: $N+2 s<4$. Let $\bar{q}=\frac{4}{4-s}, \bar{r}=2$, then we have $1 \leq \bar{q}, \bar{r} \leq 2$ and $\frac{4}{\bar{q}}-N\left(\frac{1}{2}-\frac{1}{\bar{r}}\right)=$ $4-s$. Next, we choose the admissible pair $(q, r) \in \Lambda_{b}$ that satisfy (4.24)-(4.26). We consider two subcases.

Subcase 1: $(N-2 s) \alpha<2 s-2 b$. Let

$$
q=\infty, \quad r=2 .
$$

Then it is easy to verify that $(q, r) \in \Lambda_{b}$ and (4.24)-(4.26) hold.

Subcase 2: $(N-2 s) \alpha \geq 2 s-2 b$. Let

$$
q=\frac{8(\alpha+1)}{(N-2 s) \alpha-2 s+2 b+2 \varepsilon}, \quad r=\frac{2 N(\alpha+1)}{N-2 b-2 \varepsilon+2 s(\alpha+1)},
$$

where $\varepsilon>0$ sufficiently small such that

$$
2 \varepsilon<\min \{8-2 b-(N-2 s) \alpha, N-2 b\} .
$$

Then we have $(q, r) \in \Lambda_{b}$ and

$$
\left\{\begin{array}{l}
\frac{1}{\bar{q}}-\frac{\alpha+1}{q}=\frac{8-2 b-2 \varepsilon-(N-2 s) \alpha}{8}>0, \\
\frac{N}{s}-r=\frac{N(N-2 b-2 \varepsilon)}{s(N-2 b-2 \varepsilon+2 s(\alpha+1))}>0 \\
\frac{1}{\bar{r}}-(\alpha+1)\left(\frac{1}{r}-\frac{s}{N}\right)=\frac{b+\varepsilon}{N}>\frac{b}{N} .
\end{array}\right.
$$

Hence we have that (4.24)-(4.26) hold.

Lemma 4.6. Let $N \geq 1, \beta>\max \left\{2, \frac{N}{4}\right\}, 0<s \leq 2, s<\frac{N}{2}, 0<\alpha,(N-2 s) \alpha<8-\frac{2 N}{\beta}$, $f \in \mathcal{C}(\alpha)$, and $K(x) \in L^{\infty}\left(\mathbb{R}^{N}\right)$. There exist $\sigma>0,1 \leq \bar{q}, \bar{r} \leq 2$ with $\frac{4}{\bar{q}}-N\left(\frac{1}{2}-\frac{1}{\bar{r}}\right)=4-s$ such that

$$
\left\|K \chi_{T}(f(u)-f(v))\right\|_{L^{\bar{q}} L^{\bar{r}}} \lesssim T^{\sigma}\left(\|u\|_{\mathcal{X}^{s}}^{\alpha}+\|v\|_{\mathcal{X}^{s}}^{\alpha}\right)\|u-v\|_{\mathcal{X}^{s}} .
$$

Proof. We first claim that if $1 \leq \bar{q}, \bar{r} \leq 2$ with $\frac{4}{\bar{q}}-N\left(\frac{1}{2}-\frac{1}{\bar{r}}\right)=4-s$ and $(q, r) \in \Lambda_{b}$ is an admissible pair that satisfy

$$
\left\{\begin{array}{l}
\frac{1}{\bar{q}}-\frac{\alpha+1}{q}>0, \\
r<\frac{N}{s}, \\
\frac{\alpha+1}{r}>\frac{1}{\bar{r}}>(\alpha+1)\left(\frac{1}{r}-\frac{s}{N}\right),
\end{array}\right.
$$

then the inequality (4.27) holds with $\sigma=\frac{1}{\bar{q}}-\frac{\alpha+1}{q}$. In fact, let $1<p<\infty$ be given by $\frac{1}{\bar{r}}=\frac{\alpha+1}{p}$, then by (4.30), we have $\frac{1}{r}-\frac{s}{N}<\frac{1}{p}<\frac{1}{r}$; so that the embedding $B_{r, 2}^{s}\left(\mathbb{R}^{N}\right) \hookrightarrow$ $L^{p}\left(\mathbb{R}^{N}\right)$ holds. Using (1.7), the boundedness of $K(x)$, Hölder's inequality and Sobolev's embedding $B_{r, 2}^{s}\left(\mathbb{R}^{N}\right) \hookrightarrow L^{\frac{N r}{N-s r}}\left(\mathbb{R}^{N}\right)$, we have

$$
\begin{aligned}
& \left\|K(x) \chi_{T}(t)(f(u)-f(v))\right\|_{L^{\bar{q}} L^{\bar{r}}} \\
& \lesssim\|\| K\left\|_{L^{p}}\left(\|u\|_{L^{\frac{N r}{N}-s r}}^{\alpha}+\|v\|_{L^{\frac{N r}{N}-s r}}^{\alpha}\right)\right\| u-v\left\|_{L^{\frac{N r}{N}-s r}}\right\|_{L^{\bar{q}}} \\
& \lesssim T^{\frac{1}{\bar{q}}-\frac{\alpha+1}{q}}\left(\|u\|_{L^{q} B_{r, 2}^{s}}^{\alpha}+\|v\|_{L^{q} B_{r, 2}^{s}}^{\alpha}\right)\|u-v\|_{L^{q} B_{r, 2}^{s}},
\end{aligned}
$$


which yields (4.27).

To prove Lemma 4.6, it suffices to provide $1 \leq \bar{q}, \bar{r} \leq 2$ with $\frac{4}{\bar{q}}-N\left(\frac{1}{2}-\frac{1}{\bar{r}}\right)=4-s$ and an $(q, r) \in \Lambda_{b}$ that satisfy (4.28)-(4.30). We consider three cases.

Case 1: $(N-2 s) \alpha<2 s$. Let

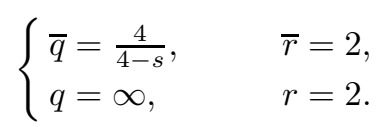

Then it is easy to verify that $1 \leq \bar{q}, \bar{r} \leq 2$ with $\frac{4}{\bar{q}}-N\left(\frac{1}{2}-\frac{1}{\bar{r}}\right)=4-s$ and an admissible pair $(q, r) \in \Lambda_{b}$ that satisfies (4.28)-4.30).

Case 2: $2 s \leq(N-2 s) \alpha<4 \alpha+4+2 s$. Let

$$
\begin{cases}\bar{q}=\frac{4}{4-s}, & \bar{r}=2, \\ q=\frac{8(\alpha+1)}{(N-2 s) \alpha-2 s+2 \varepsilon}, & r=\frac{2 N(\alpha+1)}{N-2 \varepsilon+2 s(\alpha+1)},\end{cases}
$$

where $\varepsilon>0$ sufficiently small such that

$$
\varepsilon<\min \left\{\frac{8-(N-2 s) \alpha}{2}, s(\alpha+1), \frac{N}{2}, \frac{4 \alpha+4-2 s-(N-2 s) \alpha}{2}\right\} .
$$

By direct calculation, we have $1 \leq \bar{q}, \bar{r} \leq 2$ with $\frac{4}{\bar{q}}-N\left(\frac{1}{2}-\frac{1}{\bar{r}}\right)=4-s,(q, r) \in \Lambda_{b}$ and

$$
\left\{\begin{array}{l}
\frac{1}{\bar{q}}-\frac{\alpha+1}{q}=\frac{8-(N-2 s) \alpha-2 \varepsilon}{8}>0 \\
\frac{N}{s}-r=\frac{N(N-2 \varepsilon)}{s(N-2 \varepsilon+2(\alpha+1))}>0 \\
\frac{\alpha+1}{r}-\frac{1}{\bar{r}}=\frac{s(\alpha+1)-\varepsilon}{N}>0 \\
\frac{1}{\bar{r}}-(\alpha+1)\left(\frac{1}{r}-\frac{s}{N}\right)=\frac{\varepsilon}{N}>0
\end{array}\right.
$$

which imply (4.28)-(4.30).

Case 3: $(N-2 s) \alpha>4 \alpha+4+2 s$. Let

$$
\begin{cases}\bar{q}=\frac{8(\alpha+2)}{(8-(N-2)) \alpha+16-2 \varepsilon}, & \bar{r}=\frac{N(\alpha+2)}{(N-2 s) \alpha+N-2 s+\varepsilon}, \\ q=\frac{8(\alpha+2)}{(N-2 s) \alpha+2 \varepsilon}, & r=\frac{N(\alpha+2)}{N+s \alpha-\varepsilon},\end{cases}
$$

where $\varepsilon>0$ sufficiently small such that

$$
\varepsilon<\min \left\{b, s, N-2 s, \frac{\left.8_{(} N-2 s\right) \alpha}{2}\right\} .
$$

By direct calculation, we have $1 \leq \bar{q}, \bar{r} \leq 2$ with $\frac{4}{\bar{q}}-N\left(\frac{1}{2}-\frac{1}{\bar{r}}\right)=4-s,(q, r) \in \Lambda_{b}$ and

$$
\left\{\begin{array}{l}
\frac{1}{\bar{q}}-\frac{\alpha+1}{q}=\frac{(8-2 \varepsilon-(N-2 s) \alpha)(\alpha+2)}{8(\alpha+2)}>0 \\
\frac{N}{s}-r=\frac{N(N-2 s-\varepsilon)}{s(N+s \alpha+\varepsilon)}>0 \\
\frac{\alpha+1}{r}-\frac{1}{\bar{r}}=\frac{(\alpha+2) s \alpha+s+\varepsilon}{N(\alpha+2)}>0 \\
\frac{1}{\bar{r}}-(\alpha+1)\left(\frac{1}{r}-\frac{s}{N}\right)=\frac{\varepsilon \alpha}{N(\alpha+2)}>0
\end{array}\right.
$$

Hence we see that (4.28)-(4.30) hold. 
Lemma 4.7. Let $N \geq 1, \beta>\max \left\{2, \frac{N}{4}\right\}, 0<s \leq 2, s<\frac{N}{2}, 0<\alpha,(N-2 s) \alpha<8-\frac{2 N}{\beta}$, $f \in \mathcal{C}(\alpha)$, and $K(x) \in L^{1}\left(\mathbb{R}^{N}\right) \cap L^{\beta}\left(\mathbb{R}^{N}\right)$. There exist $\sigma>0,(\gamma, \rho) \in \Lambda_{b}$ such that

$$
\left\|K \chi_{T}(f(u)-f(v))\right\|_{L^{\gamma^{\prime} L^{\rho^{\prime}}}} \lesssim T^{\sigma}\left(\|u\|_{\mathcal{X}^{s}}^{\alpha}+\|v\|_{\mathcal{X}^{s}}^{\alpha}\right) \sup _{(q, r) \in \Lambda_{b}}\|u-v\|_{L^{q} L^{r}} .
$$

Proof. Let $b=\frac{N}{\beta}$, then we have $0<b<\min \left\{\frac{N}{2}, 4\right\}$ and $(N-2 s) \alpha<8-2 b$. From the proof of Lemma 4.3, we know there exist three admissible pairs $(\gamma, \rho),(q, r),(m, n) \in \Lambda_{b}$ such that

$$
\left\{\begin{array}{l}
1-\frac{1}{\gamma}-\frac{\alpha}{q}-\frac{1}{m}>0, \\
r<\frac{N}{s} \\
1-\frac{1}{\rho}-\alpha\left(\frac{1}{r}-\frac{s}{N}\right)-\frac{1}{n}>\frac{b}{N} .
\end{array}\right.
$$

Put $\frac{1}{p}=1-\frac{1}{\rho}-\alpha\left(\frac{1}{r}-\frac{s}{N}\right)-\frac{1}{n}$, then we have $1<p<\frac{N}{b}$ and thus $K(x) \in L^{p}\left(\mathbb{R}^{N}\right)$. Using (1.7), Hölder's inequality and Sobolev's embedding $B_{r, 2}^{s}\left(\mathbb{R}^{N}\right) \hookrightarrow L^{\frac{N r}{N-s r}}\left(\mathbb{R}^{N}\right)$, we have

$$
\begin{aligned}
& \left\|K \chi_{T}(f(u)-f(v))\right\|_{L^{\gamma^{\prime}} L^{\rho^{\prime}}} \\
\lesssim & T^{1-\frac{1}{\gamma}-\frac{\alpha}{q}-\frac{1}{m}}\|K\|_{L^{p}}\left(\|u\|_{L^{q} L^{\frac{N r}{N-s r}}}^{\alpha}+\|v\|_{L^{q} L^{\frac{N r}{N-s r}}}^{\alpha}\right)\|u-v\|_{L^{m} L^{n}} \\
\lesssim & T^{1-\frac{1}{\gamma}-\frac{\alpha}{q}-\frac{1}{m}}\left(\|u\|_{L^{q} B_{r, 2}^{s}}^{\alpha}+\|v\|_{L^{q} B_{r, 2}^{s}}^{\alpha}\right)\|u-v\|_{L^{m} L^{n}},
\end{aligned}
$$

which yields (4.31).

Lemma 4.8. Let $N \geq 1, \beta>\max \left\{2, \frac{N}{4}\right\}, 0<s \leq 2, s<\frac{N}{2}, 0<\alpha,(N-2 s) \alpha<8-\frac{2 N}{\beta}$, $f \in \mathcal{C}(\alpha)$, and $K(x) \in L^{\infty}\left(\mathbb{R}^{N}\right)$. There exist $\sigma>0,(\gamma, \rho) \in \Lambda_{b}$ such that

$$
\left\|K \chi_{T}(f(u)-f(v))\right\|_{L^{\gamma^{\prime}} L^{\rho^{\prime}}} \lesssim T^{\sigma}\left(\|u\|_{\mathcal{X}^{s}}^{\alpha}+\|v\|_{\mathcal{X}^{s}}^{\alpha}\right) \sup _{(q, r) \in \Lambda_{b}}\|u-v\|_{L^{q} L^{r}} .
$$

Proof. From the proof of Lemma 4.4, we know there exists $(q, r) \in \Lambda_{b}$ such that

$$
\left\{\begin{array}{l}
1-\frac{\alpha+2}{q}>0, \\
r<\frac{N}{s} \\
\frac{\alpha}{r}+\frac{1}{r}>1-\frac{1}{r}>\alpha\left(\frac{1}{r}-\frac{s}{N}\right)+\frac{1}{r} .
\end{array}\right.
$$

Let $p$ be given by $1-\frac{1}{r}=\frac{\alpha}{p}+\frac{1}{r}$, then we have $\frac{1}{r}-\frac{s}{N}<\frac{1}{p}<\frac{1}{r}$ and thus the embedding $B_{r, 2}^{s}\left(\mathbb{R}^{N}\right) \hookrightarrow L^{p}\left(\mathbb{R}^{N}\right)$ holds. Using (1.7), the boundedness of $K(x)$, Hölder's inequality and Sobolev's embedding $B_{r, 2}^{s}\left(\mathbb{R}^{N}\right) \hookrightarrow L^{p}\left(\mathbb{R}^{N}\right)$, we have

$$
\left\|K \chi_{T}(f(u)-f(v))\right\|_{L^{q^{\prime}} L^{r^{\prime}}} \lesssim T^{1-\frac{\alpha+2}{q}}\left(\|u\|_{L^{q} B_{r, 2}^{s}}^{\alpha}+\|v\|_{L^{q} B_{r, 2}^{s}}^{\alpha}\right)\|u-v\|_{L^{q} L^{r}},
$$

which yields (4.32). 
In the case $s \geq \frac{N}{2}$, the embedding $H^{s}\left(\mathbb{R}^{N}\right) \hookrightarrow L^{p}\left(\mathbb{R}^{N}\right)$ holds for any $2 \leq p<\infty$; thus we can establish the following Lemmas.

Lemma 4.9. Let $N \geq 1, \beta>\max \left\{2, \frac{N}{4}\right\}, 0<s \leq 2, s \geq \frac{N}{2}, 0<\alpha<\infty, f \in \mathcal{C}(\alpha)$, and $K(x) \in L^{\infty}\left(\mathbb{R}^{N}\right)+L^{\beta}\left(\mathbb{R}^{N}\right)$. There exist $\sigma>0$ and $2 \leq r, \rho<\infty$ such that

$$
\begin{aligned}
& \left(\int_{-\infty}^{\infty}\left(|\tau|^{-s / 4}\left\|\left(\chi_{T}(t-\tau)-\chi_{T}(\tau)\right) K(x)(f(u)-f(v))\right\|_{L^{1} L^{2}}\right)^{2} \frac{\mathrm{d} \tau}{|\tau|}\right)^{1 / 2} \\
& \lesssim T^{\sigma}\left(\|u\|_{\mathcal{X}^{s}}^{\alpha}+\|v\|_{\mathcal{X}^{s}}^{\alpha}\right)\|u-v\|_{\mathcal{X}^{s}},
\end{aligned}
$$

and

$$
\begin{aligned}
& \left(\int_{-\infty}^{\infty}\left(|\tau|^{-s / 4}\left\|\chi_{T}(t-\tau) K(x)\left((f(u)-f(v))_{\tau}-(f(u)-f(v))\right)\right\|_{L^{1} L^{2}}\right)^{2} \frac{\mathrm{d} \tau}{|\tau|}\right)^{1 / 2} \\
& \lesssim T^{\sigma}\left(\|u\|_{\mathcal{X}^{s}}^{\alpha}+\|v\|_{\mathcal{X}^{s}}^{\alpha}\right)\|u-v\|_{\mathcal{X}^{s}}+1_{\alpha<1}\|u-v\|_{L^{\infty}\left(\mathbb{R}, L^{r} \cap L^{\rho}\right)}^{\alpha}\|v\|_{\mathcal{X}^{s}} .
\end{aligned}
$$

Proof. Since $K(x) \in L^{\infty}\left(\mathbb{R}^{N}\right)+L^{\beta}\left(\mathbb{R}^{N}\right) \subset L^{\infty}\left(\mathbb{R}^{N}\right)+L^{1}\left(\mathbb{R}^{N}\right) \cap L^{\beta}\left(\mathbb{R}^{N}\right)$, we can decompose $K(x)=K_{1}(x)+K_{2}(x)$, with $K_{1}(x) \in L^{\infty}\left(\mathbb{R}^{N}\right), K_{2}(x) \in L^{1}\left(\mathbb{R}^{N}\right) \cap L^{\beta}\left(\mathbb{R}^{N}\right)$. Put $(q, r)$, $(\gamma, \rho) \in \Lambda_{b}$

$$
\begin{cases}q=\frac{8(\alpha+1)(2+\varepsilon)}{N(2 \alpha+2+\varepsilon \alpha)}, & r=\frac{2(2+\varepsilon)(\alpha+1)}{\varepsilon}, \\ \gamma=\frac{8(\alpha+1)}{N \alpha}, & \rho=2 \alpha+2,\end{cases}
$$

where $0<\varepsilon<\beta-2$; so that $K_{2}(x) \in L^{1}\left(\mathbb{R}^{N}\right) \cap L^{\beta}\left(\mathbb{R}^{N}\right) \subset L^{2+\varepsilon}\left(\mathbb{R}^{N}\right)$.

We first prove (4.34). From (4.16), (4.17) and (4.18), the boundedness of $\chi_{T}$ and Hölder's inequality, we get

$$
\begin{aligned}
& \left\|\chi_{T}(t-\tau) K_{2}(x)\left((f(u)-f(v))_{\tau}-(f(u)-f(v))\right)\right\|_{L^{1} L^{2}} \\
\lesssim & T^{1-\frac{1}{q}}\left\|(u-v)_{\tau}-(u-v)\right\|_{L^{q} L^{r}}\left\|K_{2}(x)\right\|_{L^{2+\varepsilon}}\left(\|u\|_{L^{\infty} L^{r}}^{\alpha}+\|v\|_{L^{\infty} L^{r}}^{\alpha}\right) \\
& +T^{1-\frac{1}{q}}\left\{\begin{array}{l}
\left\|v_{\tau}-v\right\|_{L^{q} L^{r}}\left(\|u\|_{L^{\infty} L^{r}}^{\alpha-1}+\|v\|_{L^{\infty} L^{r}}^{\alpha-1}\right)\|u-v\|_{L^{q} L^{r}}, \alpha \geq 1, \\
\left\|v_{\tau}-v\right\|_{L^{q} L^{r}}\|u-v\|_{L^{\infty} L^{r}}^{\alpha}, 0<\alpha<1 .
\end{array}\right.
\end{aligned}
$$

This inequality together with young's inequality yields

$$
\begin{aligned}
& \left(\int_{-\infty}^{\infty}\left(|\tau|^{-s / 4}\left\|\chi_{T}(t-\tau) K_{2}(x)\left((f(u)-f(v))_{\tau}-(f(u)-f(v))\right)\right\|_{L^{1} L^{2}}\right)^{2} \frac{\mathrm{d} \tau}{|\tau|}\right)^{1 / 2} \\
& \lesssim T^{1-\frac{1}{q}}\left(\|u\|_{\mathcal{X}^{s}}^{\alpha}+\|v\|_{\mathcal{X}^{s}}^{\alpha}\right)\|u-v\|_{\mathcal{X}^{s}}+1_{\alpha<1}\|u-v\|_{L^{\infty} L^{r}}^{\alpha}\|v\|_{\mathcal{X}^{s}} .
\end{aligned}
$$

Similarly, we have

$$
\begin{aligned}
& \left(\int_{-\infty}^{\infty}\left(|\tau|^{-s / 4}\left\|\chi_{T}(t-\tau) K_{1}(x)\left((f(u)-f(v))_{\tau}-(f(u)-f(v))\right)\right\|_{L^{1} L^{2}}\right)^{2} \frac{\mathrm{d} \tau}{|\tau|}\right)^{1 / 2} \\
& \lesssim T\left(\|u\|_{\mathcal{X}^{s}}^{\alpha}+\|v\|_{\mathcal{X}^{s}}^{\alpha}\right)\|u-v\|_{\mathcal{X}^{s}}+1_{\alpha<1}\|u-v\|_{L^{\infty} L^{\rho}}^{\alpha}\|v\|_{\mathcal{X}^{s}} .
\end{aligned}
$$

The inequality (4.34) is now an immediate consequence of (4.35) and (4.36).

Next, we prove (4.33). From (1.7) and Hölder's inequality, we have

$$
\begin{aligned}
& \left\|\left(\chi_{T}(t-\tau)-\chi_{T}(t)\right) K_{2}(f(u)-f(v))\right\|_{L^{1} L^{2}} \\
\lesssim & \left\|\chi_{T}(t-\tau)-\chi_{T}(t)\right\|_{L^{1}}\left\|K_{2}\right\|_{L^{2+\varepsilon}}\left(\|u\|_{L^{\infty} L^{r}}^{\alpha}+\|v\|_{L^{\infty} L^{r}}^{\alpha}\right)\|u-v\|_{L^{\infty} L^{r}} .
\end{aligned}
$$


This inequality together with Sobolev's embedding $H^{s}\left(\mathbb{R}^{N}\right) \hookrightarrow L^{r}\left(\mathbb{R}^{N}\right)$ yields

$$
\begin{aligned}
& \left(\int_{-\infty}^{\infty}\left(|\tau|^{-s / 4}\left\|\left(\chi_{T}(t-\tau)-\chi_{T}(\tau)\right) K_{2}(x)(f(u)-f(v))\right\|_{L^{1} L^{2}}\right)^{2} \frac{\mathrm{d} \tau}{|\tau|}\right)^{1 / 2} \\
& \lesssim T^{1-\frac{s}{4}}\left(\|u\|_{\mathcal{X}^{s}}^{\alpha}+\|v\|_{\mathcal{X}^{s}}^{\alpha}\right)\|u-v\|_{\mathcal{X}^{s}},
\end{aligned}
$$

where we used $\left\|\chi_{T}\right\|_{B_{1,2}^{s / 4}} \lesssim T^{1-\frac{s}{4}}$. Similarly, we have

$$
\begin{aligned}
& \left(\int_{-\infty}^{\infty}\left(|\tau|^{-s / 4}\left\|\left(\chi_{T}(t-\tau)-\chi_{T}(\tau)\right) K_{1}(x)(f(u)-f(v))\right\|_{L^{1} L^{2}}\right)^{2} \frac{\mathrm{d} \tau}{|\tau|}\right)^{1 / 2} \\
& \lesssim T\left(\|u\|_{\mathcal{X}^{s}}^{\alpha}+\|v\|_{\mathcal{X}^{s}}^{\alpha}\right)\|u-v\|_{\mathcal{X}^{s}} .
\end{aligned}
$$

The inequality (4.33) is now an immediate consequence of (4.37) and (4.38).

Similar to Lemma 4.9, we can establish the following Lemma and omit the details.

Lemma 4.10. Let $N \geq 1, \beta>\max \left\{2, \frac{N}{4}\right\}, 0<s \leq 2, s \geq \frac{N}{2}, 0<\alpha<\infty, f \in \mathcal{C}(\alpha)$, and $K(x) \in L^{\infty}\left(\mathbb{R}^{N}\right)+L^{\beta}\left(\mathbb{R}^{N}\right)$. There exist $\sigma>0$ such that

$$
\left\|K \chi_{T}(f(u)-f(v))\right\|_{L^{\bar{q}} L^{T}} \lesssim T^{\sigma}\left(\|u\|_{\mathcal{X}^{s}}^{\alpha}+\|v\|_{\mathcal{X}^{s}}^{\alpha}\right)\|u-v\|_{\mathcal{X}^{s}},
$$

where $\bar{q}=\frac{4}{4-s}, \bar{r}=2$ with $1 \leq \bar{q}, \bar{r} \leq 2, \frac{4}{\bar{q}}-N\left(\frac{1}{2}-\frac{1}{\bar{r}}\right)=4-s$ and

$$
\left\|K \chi_{T}(f(u)-f(v))\right\|_{L^{1} L^{2}} \lesssim T^{\sigma}\left(\|u\|_{\mathcal{X}^{s}}^{\alpha}+\|v\|_{\mathcal{X}^{s}}^{\alpha}\right) \sup _{(q, r) \in \Lambda_{b}}\|u-v\|_{L^{q} L^{r}} .
$$

\section{Proof of Theorem 1.5 and Theorem 1.9}

\subsection{The local existence}

Since $K(x) \in L^{\infty}\left(\mathbb{R}^{N}\right)+L^{\beta}\left(\mathbb{R}^{N}\right) \subset L^{\infty}\left(\mathbb{R}^{N}\right)+L^{1}\left(\mathbb{R}^{N}\right) \cap L^{\beta}\left(\mathbb{R}^{N}\right)$, we can decompose $K(x)=K_{1}(x)+K_{2}(x)$, with $K_{1}(x) \in L^{\infty}\left(\mathbb{R}^{N}\right), K_{2}(x) \in L^{1}\left(\mathbb{R}^{N}\right) \cap L^{\beta}\left(\mathbb{R}^{N}\right)$. Then using Lemmas 4.14 .10 and the standard contraction mapping argument, we can establish the following local existence results easily and omit the details.

Proposition 5.1. Let $N \geq 1, \mu=-1$ or $0, \beta>\max \left\{2, \frac{N}{4}\right\}, 0<s \leq 2,0<\alpha$, $(N-2 s) \alpha<8-\frac{2 N}{\beta}, f \in \mathcal{C}(\alpha)$, and $K(x) \in L^{\infty}\left(\mathbb{R}^{N}\right)+L^{\beta}\left(\mathbb{R}^{N}\right)$. There exist constants $C_{1}>0$ and $c(L)>0$ that depends only on $L$ such that for any $L \geq 2 C_{1}\|\varphi\|_{H^{s}}, 0<T \leq$ $c(L)$, the equation

$$
u=e^{i t\left(\Delta^{2}+\mu \Delta\right)} \varphi-i \int_{0}^{t} e^{i(t-\tau)\left(\Delta^{2}+\mu \Delta\right)}\left(\chi_{T} K f(u)\right)(s) \mathrm{d} \tau
$$

admits a unique solution $u \in C\left(\mathbb{R}, H^{s}\left(\mathbb{R}^{N}\right)\right) \cap \mathcal{X}^{s}$ with $\|u\|_{\mathcal{X}^{s}} \leq L$.

Since $\left.\chi_{T}\right|_{t \in[0, T]}=1$, it follows from Proposition 5.1 that there exists a solution $u \in$ $C\left([0, T], H^{s}\right) \bigcap_{(q, r) \in \Lambda_{b}} L^{q}\left([0, T], B_{r, 2}^{s}\right)$ to the Cauchy problem (1.1). Moreover, using the similar arguments as that in Chapter 4 of [6], we deduce that the Cauchy problem (1.1) admits a unique maximum solution

$$
u \in C\left(\left[0, T_{\max }(\varphi)\right), H^{s}\right) \bigcap_{(q, r) \in \Lambda_{b}} L^{q}\left(\left[0, T_{\max }(\varphi)\right), B_{r, 2}^{s}\right)
$$

with the blowup alternative (1.8) holds, and omit the details. 


\subsection{The continuous dependence}

In this subsection, we prove continuous dependence of the solution map on the interval $[0, A]$, where $0<A<T_{\max }(\varphi)$. Let

$$
L=4 C_{1}\|u\|_{L^{\infty}\left([0, T], H^{s}\right)}<\infty,
$$

and $T>0$ sufficiently small such that

$$
T \leq c(L), \quad C_{2} T^{\widetilde{\sigma}} L^{\alpha}+C_{3} T^{\sigma} L^{\alpha} \leq \frac{1}{4},
$$

where $C_{1}, c(L)$ are the constants in Proposition 5.1, $C_{2}, \widetilde{\sigma}$ and $C_{3}, \sigma$ are the constants in (5.6) and (5.7), respectively. Since $\varphi_{n} \rightarrow \varphi$ in $H^{s}$, we know that there exists a positive $n_{1}$ such that $L \geq 2 C_{1}\left\|\varphi_{n}\right\|_{H^{s}}$ for every $n \geq n_{1}$. It then follows from Proposition 5.1 that for every $n \geq n_{1}$, the following equation

$$
u_{n}=e^{i t\left(\Delta^{2}+\mu \Delta\right)} \varphi_{n}-i \int_{0}^{t} e^{i(t-\tau)\left(\Delta^{2}+\mu \Delta\right)}\left(\chi_{T} K f\left(u_{n}\right)\right)(\tau) \mathrm{d} \tau,
$$

admits a unique solution $u_{n} \in C\left(\mathbb{R}, H^{s}\right) \cap \mathcal{X}^{s}$ with

$$
\left\|u_{n}\right\|_{\mathcal{X}^{s}} \leq L, \text { for } \forall n \geq n_{1} .
$$

Moreover, Proposition 5.1 also gives us a unique solution $v \in C\left(\mathbb{R}, H^{s}\right) \cap \mathcal{X}^{s}$ to the equation (5.1) that satisfies

$$
\|v\|_{\mathcal{X}^{s}} \leq L
$$

The proof of the continuous dependence will proceed by the following claims.

Claim 5.1. For any $0<\delta<s$, we have $\sup _{(q, r) \in \Lambda_{b}}\left\|u_{n}-v\right\|_{L^{q}\left(\mathbb{R}, B_{r, 2}^{s-\delta}\right)} \underset{n \rightarrow \infty}{\longrightarrow} 0$..

Proof. Let $K(x)=K_{1}(x)+K_{2}(x)$ with $K_{1}(x) \in L^{\beta}\left(\mathbb{R}^{N}\right)$ and $K_{2}(x) \in L^{\infty}\left(\mathbb{R}^{N}\right)$. From Lemmas 4.7 4.8 and 4.10, we conclude that there exist $\left(\gamma_{i}, \rho_{i}\right) \in \Lambda_{b}, \sigma_{i}>0, i=1,2$, such that

$$
\begin{aligned}
& \left\|K_{i} \chi_{T}(f(u)-f(v))\right\|_{L^{\gamma_{i}^{\prime} L^{\rho_{i}^{\prime}}}}\left\|T^{\sigma_{i}}\left(\|u\|_{\mathcal{X}^{s}}^{\alpha}+\|v\|_{\mathcal{X}^{s}}^{\alpha}\right) \sup _{(q, r) \in \Lambda_{b}}\right\| u-v \|_{L^{q} L^{r}}, i=1,2 .
\end{aligned}
$$

From Strichartz's estimate and (5.5), we deduce that there exists $C_{2}>0$ such that

$$
\begin{aligned}
& \sup _{(q, r) \in \Lambda_{b}}\left\|u_{n}-v\right\|_{L^{q} L^{r}} \\
\leq & C_{2}\left\|\varphi_{n}-\varphi\right\|_{L^{2}}+C_{2} T^{\widetilde{\sigma}}\left(\left\|u_{n}\right\|_{\mathcal{X}^{s}}^{\alpha}+\|v\|_{\mathcal{X}^{s}}^{\alpha}\right) \sup _{(q, r) \in \Lambda_{b}}\left\|u_{n}-v\right\|_{L^{q} L^{r}},
\end{aligned}
$$

where $\widetilde{\sigma}=\min \left\{\sigma_{1}, \sigma_{2}\right\}$. Since $2 C_{2} T^{\widetilde{\sigma}} L^{\alpha} \leq \frac{1}{2}$ by (5.2), it follows from (5.3), (5.4) and (5.6) that

$$
\sup _{(q, r) \in \Lambda_{b}}\left\|u_{n}-v\right\|_{L^{q}\left(\mathbb{R}, L^{r}\right)} \lesssim\left\|\varphi_{n}-\varphi\right\|_{L^{2}} .
$$

Moreover, from the interpolation theorem, (5.3) and (5.4), we have

$$
\begin{aligned}
\sup _{(q, r) \in \Lambda_{b}}\left\|u_{n}-v\right\|_{L^{q}\left(\mathbb{R}, B_{r, 2}^{s-\delta}\right)} & \lesssim\left\|u_{n}-v\right\|_{L^{q}\left(\mathbb{R}, B_{r, 2}^{s}\right)}^{1-\frac{\delta}{s}}\left\|u_{n}-v\right\|_{L^{q}\left(\mathbb{R}, L^{r}\right)}^{\frac{\delta}{s}} \\
& \lesssim L^{1-\frac{\delta}{s}}\left\|\varphi_{n}-\varphi\right\|_{L^{2}}^{\frac{\delta}{s}} \underset{n \rightarrow \infty}{\longrightarrow} 0,
\end{aligned}
$$

which finishes the proof of Claim 5.1 . 
Claim 5.2. $\left\|u_{n}-v\right\|_{\mathcal{X}^{s}} \underset{n \rightarrow \infty}{\longrightarrow} 0$.

Proof. When $s<\frac{N}{2}$, we deduce from Proposition 1.1, Lemmas 4.14.8 that, for some $C_{3}, \sigma>0$,

$$
\begin{aligned}
\left\|u_{n}-v\right\|_{\mathcal{X}^{s}} \leq & C_{3}\left\|\varphi_{n}-\varphi\right\|_{H^{s}}+C_{3} T^{\sigma}\left(\left\|u_{n}\right\|_{\mathcal{X}^{s}}^{\alpha}+\|v\|_{\mathcal{X}^{s}}^{\alpha}\right)\left\|u_{n}-v\right\|_{\mathcal{X}^{s}} \\
& +1_{\alpha<1} C_{3}\left\|u_{n}-v\right\|_{L_{\mathrm{uloc}, T}^{\widetilde{1_{1}}}}^{\alpha} L^{\frac{N r_{1}}{N-s r_{1}} \cap L_{\mathrm{uloc}, T}^{\widetilde{q_{2}}} L^{\frac{N r_{2}}{N-s r_{2}}}\|v\|_{\mathcal{X}^{s}}},
\end{aligned}
$$

where $\widetilde{q}_{i}>1,\left(q_{i}, r_{i}\right) \in \Lambda_{b}$ with $\widetilde{q}_{i}<q_{i}, r_{i}<4^{*}, i=1,2$ are given by Lemmas 4.3 and 4.4 , respectively. Since $2 C_{3} T^{\sigma} L^{\alpha} \leq \frac{1}{2}$ by (5.2), we deduce from (5.3), (5.4) and (5.7) that

$$
\left\|u_{n}-v\right\|_{\mathcal{X}^{s}} \lesssim\left\|\varphi_{n}-\varphi\right\|_{H^{s}}+\left\|u_{n}-v\right\|_{L_{\mathrm{uloc}, T}^{\widetilde{q_{1}}}}^{\alpha} L^{\frac{N r_{1}}{N-s r_{1}} \cap L_{\mathrm{uloc}, T}^{\widetilde{q_{2}}} L^{\frac{N r_{2}}{N-s r_{2}}}} \cdot
$$

Thus it suffices to show that

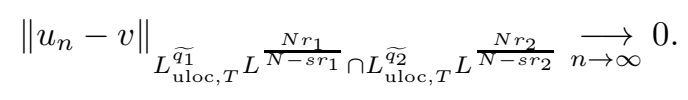

For any $\eta>0$ sufficiently small such that $r_{1}+\eta<4^{*}$, we choose $q_{\eta}>0$ such that $\left(q_{\eta}, r_{1}+\eta\right) \in \Lambda_{b}$. From Sobolev embedding $B_{r_{1}+\eta, 2}^{s-\frac{\eta N}{r_{1}\left(r_{1}+\eta\right)}}\left(\mathbb{R}^{N}\right) \hookrightarrow L^{\frac{N r_{1}}{N-s r_{1}}}\left(\mathbb{R}^{N}\right)$ and the claim [5.1] we have

$$
\left\|u_{n}-v\right\|_{L_{\mathrm{uloc}, T}^{q_{\eta}} L^{\frac{N r_{1}}{N-s r_{1}}}} \lesssim\left\|u_{n}-v\right\|_{L^{q_{\eta}} B_{r_{1}+\eta, 2}^{s-\frac{\eta N}{r_{1}\left(r_{1}+\eta\right)}}}^{\underset{n \rightarrow \infty}{\longrightarrow}} 0 .
$$

Since $q_{\eta}$ increasing to $q_{1}$ as $\eta$ tends to 0 and $\widetilde{q_{1}}<q_{1}$, we have, by applying Hölder's inequality

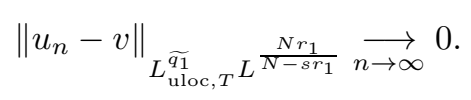

Similarly, we have

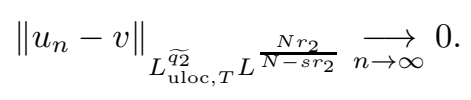

This together with (5.10) yields (5.8).

We now consider the case $s \geq \frac{N}{2}$. In this case, we use Lemmas 4.9 and 4.10 instead of Lemmas 4.14.8, Similar to (5.8), it suffices to prove that

$$
\left\|u_{n}-v\right\|_{L^{\infty}\left(\mathbb{R}, L^{r} \cap L^{\rho}\right)} \underset{n \rightarrow \infty}{\longrightarrow} 0,
$$

where $2 \leq r, \rho<\infty$ are given by Lemma 4.9. Let $p=r+\rho$, then by interpolation theorem, there exists $0 \leq \theta<1$ such that

$$
\left\|u_{n}-v\right\|_{L^{\infty} L^{r}} \lesssim\left\|u_{n}-v\right\|_{L^{\infty} L^{p}}^{\theta}\left\|u_{n}-v\right\|_{L^{\infty} L^{2}}^{1-\theta} .
$$

From (5.11), Sobolev's embedding $H^{s}\left(\mathbb{R}^{N}\right) \hookrightarrow L^{p}\left(\mathbb{R}^{N}\right)$, (5.3), (5.4) and Claim 5.1, we have

$$
\left\|u_{n}-v\right\|_{L^{\infty} L^{r}} \underset{n \rightarrow \infty}{\longrightarrow} 0
$$

Similar, we have

$$
\left\|u_{n}-v\right\|_{L^{\infty} L^{\rho}} \underset{n \rightarrow \infty}{\longrightarrow} 0 .
$$

This finishes the proof of Claim 5.2 . 
Now we resume the proof of the continuous dependence. Since $\left.\chi_{T}\right|_{t \in[0, T]}=1$, we deduce from the uniqueness that $u(t)=v(t)$ on the interval $[0, T]$. Moreover, it follows from Claim 5.2 that $\left\|u_{n}-u\right\|_{L^{\infty}\left([0, T], H^{s}\right)} \underset{n \rightarrow \infty}{\longrightarrow} 0$. In particular, we have $\left\|u_{n}(T)-u(T)\right\|_{H^{s}} \underset{n \rightarrow \infty}{\longrightarrow} 0$. Arguing as previously, we deduce that the solution $u_{n}$ exists on the interval $[T, 2 T]$ for $n \geq n_{2}$ and that $\left\|u_{n}-u\right\|_{L^{\infty}\left([T, 2 T], H^{s}\right)} \underset{n \rightarrow \infty}{\longrightarrow} 0$. Iterating finitely many times like this, we get the continuous dependence on the interval $[0, A]$.

\subsection{Proof of Theorem 1.9}

Before proving Proposition 1.1, we prove the following version of the Caffarelli-KohnNirenberg-type inequality.

Lemma 5.2. Let $N \geq 1, \beta>\max \left\{2, \frac{N}{4}\right\}, 0<\alpha,(N-4) \alpha<8-\frac{2 N}{\beta}, f \in \mathcal{C}(\alpha)$, and $K(x) \in L^{\infty}\left(\mathbb{R}^{N}\right)+L^{\beta}\left(\mathbb{R}^{N}\right)$. Then, we have

$$
\int_{\mathbb{R}^{N}}|K(x) f(u) u| d x \lesssim\|\Delta u\|_{L^{2}}^{\frac{N(\beta \alpha+2)}{4 \beta}}\|u\|_{L^{2}}^{\alpha+2-\frac{N(\beta \alpha+2)}{4 \beta}}+\|\Delta u\|_{L^{2}}^{\frac{N \alpha}{4}}\|u\|_{L^{2}}^{\alpha+2-\frac{N \alpha}{4}} .
$$

Proof. Let $K(x)=K_{1}(x)+K_{2}(x)$ with $K_{1}(x) \in L^{\infty}\left(\mathbb{R}^{N}\right)$ and $K_{2}(x) \in L^{1}\left(\mathbb{R}^{N}\right) \cap L^{\beta}\left(\mathbb{R}^{N}\right)$. From (1.7), Hölder's inequality and Gagliardo-Nirenberg's inequality, we have

$$
\int_{\mathbb{R}^{N}}\left|K_{2}(x) f(u) u\right| d x \lesssim\left\|K_{2}\right\|_{L^{\beta}}\|u\|_{L^{(\alpha+2) \beta^{\prime}}}^{\alpha+2} \lesssim\|\Delta u\|_{L^{2}}^{\frac{N(\beta \alpha+2)}{4 \beta}}\|u\|_{L^{2}}^{\alpha+2-\frac{N(\beta \alpha+2)}{4 \beta}} .
$$

Similarly, we have

$$
\int_{\mathbb{R}^{N}}\left|K_{1}(x) f(u) u\right| d x \lesssim\|\Delta u\|_{L^{2}}^{\frac{N \alpha}{4}}\|u\|_{L^{2}}^{\alpha+2-\frac{N \alpha}{4}} .
$$

The inequality (5.12) is now an immediate consequence of (5.13) and (5.14).

Using the classical energy estimate method, we can obtain the following conservation law for the Cauchy problem (1.1) easily and omit the details.

Lemma 5.3. Let $N \geq 1, \mu=-1$ or $0, \beta>\max \left\{2, \frac{N}{4}\right\}, 0<\alpha,(N-4) \alpha<8-\frac{2 N}{\beta}$, $K(x) \in L^{\infty}\left(\mathbb{R}^{N}\right)+L^{\beta}\left(\mathbb{R}^{N}\right)$ be a real-valued function and $f \in \mathcal{C}(\alpha)$ that satisfies

(i) $f(a) \in \mathbb{R}$ for all $a \geq 0$;

(ii) $f(u)=\frac{u}{|u|} f(|u|)$ for all $u \in \mathbb{C} \backslash\{0\}$;

If $u$ is a smooth solution of (1.1) on the time interval [0,T], then the mass and the energy are conserved:

$$
\begin{gathered}
M[u](t)=\int_{\mathbb{R}^{N}}|u|^{2} d x=M[\varphi], \\
E(u)(t)=\int_{\mathbb{R}^{N}} \frac{1}{2}\left(|\Delta u|^{2}-\mu|\nabla u|^{2}\right) d x+\int_{\mathbb{R}^{N}} K(x) G(u(x)) d x=E[\phi],
\end{gathered}
$$

where $G(z):=\int_{0}^{|z|} f(s) \mathrm{d} s$.

Proof of Theorem 1.9, Let $u \in C\left(\left[0, T_{\max }(\varphi)\right), H^{2}\left(\mathbb{R}^{N}\right)\right)$ be the maximum solution given by Theorem 1.5. To obtain a global solution, it is sufficient to get an a priori bound of the local solution, since the existence time obtained in Corollary 1.6 depends only the $H^{2}$ norm of the initial datum. 
Note that $|G(u(x))| \lesssim|f(u) u|$, we deduce from Gagliardo-Nirenberg's inequality, Lemma [5.2, and the conservation of the energy (5.16) that, for any $t \in\left[0, T_{\max }(\varphi)\right)$,

$$
\begin{aligned}
\|\Delta u(t)\|_{L^{2}}^{2}= & 2 E[\phi]+\mu\|\nabla u(t)\|_{2}^{2}-2 \int_{\mathbb{R}^{N}} K(x) G(u(x)) d x \\
\leq & 2 E[\phi]+\|u(t)\|_{L^{2}}\|\Delta u(t)\|_{L^{2}}+C\|\Delta u\|_{L^{2}}^{\frac{N \alpha}{4}}\|u\|_{L^{2}}^{\alpha+2-\frac{N \alpha}{4}} \\
& +\|\Delta u\|_{L^{2}}^{\frac{N(\beta \alpha+2)}{4 \beta}}\|u\|_{L^{2}}^{\alpha+2-\frac{N(\beta \alpha+2)}{4 \beta}}
\end{aligned}
$$

From the conservation of the mass (5.15) and Cauchy-Schwartz's inequality, we have, for any $t \in\left[0, T_{\max }(\varphi)\right)$,

$$
\|u(t)\|_{L^{2}}\|\Delta u(t)\|_{L^{2}} \leq \frac{1}{4}\|\Delta u(t)\|_{L^{2}}^{2}+\|\varphi\|_{L^{2}}^{2} .
$$

On the other hand, from Young's inequality and the conservation of the mass (5.15), we have

$$
C\|\Delta u(t)\|_{L^{2}}^{\frac{\alpha N}{4}}\|\varphi\|_{L^{2}}^{\alpha+2-\frac{\alpha N}{4}} \leq \frac{1}{4}\|\Delta u\|_{L^{2}}^{2}+C^{2}\|\varphi\|_{L^{2}}^{\left(\alpha+2-\frac{\alpha N}{4}\right) \frac{8}{8-\alpha N}} .
$$

Suppose $\alpha<\frac{8}{N}-\frac{2}{\beta}$. Since $\frac{N(\beta \alpha+2)}{4 \beta}<2$, we have

$$
C\|\Delta u\|_{L^{2}}^{\frac{N(\beta \alpha+2)}{4 \beta}}\|u\|_{L^{2}}^{\alpha+2-\frac{N(\beta \alpha+2)}{4 \beta}} \leq \frac{1}{4}\|\Delta u\|_{L^{2}}^{2}+C^{2}\|\phi\|_{L^{2}}^{\left(\alpha+2-\frac{N(\beta \alpha+2)}{4 \beta}\right) \frac{8 \beta}{(8-N \alpha) \beta-2 N}} .
$$

From the above two inequalities, (5.17) -(5.20) and the conservation of the mass (5.15), we conclude that $\sup _{t \in\left[0, T_{\max }(\varphi)\right)}\|u(t)\|_{H^{2}}<\infty$, which in turn implies the global existence.

If $\alpha=\frac{8}{N}-\frac{2}{\beta}$, we deduce from (5.17), (5.18) and (5.19) that

$$
\left(\frac{1}{2}-C\|\varphi\|_{L^{2}}^{\alpha}\right)\|\Delta u(t)\|_{L^{2}}^{2} \leq 2 E[\phi]+\|\varphi\|_{L^{2}}^{2}+\|\varphi\|_{L^{2}}^{\left(\alpha+2-\frac{\alpha N}{4}\right) \frac{8}{8-\alpha N}} .
$$

Hence, the Laplacian of $u$ remains bounded if $\|\phi\|_{L^{2}} \leq\left(\frac{1}{2 C}\right)^{1 / \alpha}$, which completes the proof of Theorem 1.9.

\section{Acknowledgments}

This work is partially supported by the National Natural Science Foundation of China 11771389,11931010 and 11621101.

\section{References}

[1] J. An, J. Kim, Local well-posedness for the inhomogeneous nonlinear Schrödinger equation in $H^{s}\left(\mathbb{R}^{n}\right)$, Nonlinear Anal. Real World Appl., 59 (2021), 103268.

[2] L. Bergé, Soliton stability versus collapse, Phys. Rev. E, 62 (2000), 3071-3074.

[3] O.V. Borovkova, Y.V. Kartashov, B.A. Malomed, L. Torner, Algebraic bright and vortex solitons in defocusing media, Opt. Lett., 3616 (2011), 3088-3090. 
[4] O.V. Borovkova, Y.V. Kartashov, V.A. Vysloukh, V.E. Lobanov, B.A. Malomed, L. Torner, Solitons supported by spatially inhomogeneous nonlinear losses, Opt. Express, 203 (2012), 2657-2667.

[5] M. Cardoso, C. Guzmán, A. Pastor, Global well-posedness and critical norm concentration for inhomogeneous biharmonic NLS, (2020) arXiv:2011.04715v1.

[6] T. Cazenave, Semilinear Schrödinger equations, Courant Lecture Notes in Mathematics, American Mathematical Society, New York; Providence, RI, (2003).

[7] S. Cui, C. Guo, Well-posedness of higher-order nonlinear Schrödinger equations in Sobolev spaces $H^{s}\left(\mathbb{R}^{N}\right)$ and applications, Nonlinear Anal., 67 (2007), 687-707.

[8] V.D. Dinh, Scattering theory in weighted $L^{2}$ space for a class of the defocusing inhomogeneous nonlinear Schrödinger equation, (2020) arXiv:1710.01392v2.

[9] V.D. Dinh, On well-posedness, regularity and ill-posedness for the nonlinear fourthorder Schrödinger equation, Bull. Belg. Math. Soc. Simon Stevin, 25 (2018), 415-437.

[10] L.G. Farah, Global well-posedness and blow-up on the energy space for the inhomogeneous nonlinear Schrödinger equation, J. Evol. Equ., 16 (2016), 193-208.

[11] G. Fibich, X.P. Wang, Stability of solitary waves for nonlinear Schrödinger equations with inhomogeneous nonlinearities, Physica D, 175 (1-2) (2003), 96-108.

[12] F. Genoud, An inhomogeneous, $L^{2}$-critical, nonlinear Schrödinger equation, Z. Anal. Anwend., 31 (2012), 283-290.

[13] F. Genoud, C.A. Stuart, Schrödinger equations with a spatially decaying nonlinearity: existence and stability of standing waves, Discrete Contin. Dyn. Syst., 21 (2008), $137-186$.

[14] T.S. Gill, Optical guiding of laser beam in nonuniform plasma, Pramana J. Phys., 55(5-6) (2000), 835-842.

[15] C.M. Guzmán, On well posedness for the inhomogeneous nonlinear Schrödinger equation, Nonlinear Anal. Real World Appl., 37 (2017), 249-286.

[16] C.M. Guzmán, A. Pastor, On the inhomogeneous biharmonic nonlinear Schrödinger equation: Local, global and stability results, Nonlinear Anal. Real World Appl., 56 (2020), 103174.

[17] J. Holmer, S. Roudenko, A sharp condition for scattering of the radial $3 D$ cubic nonlinear Schrödinger equation, Comm. Math. Phys., 282 (2008), 435-467.

[18] Y. Liu, X.P. Wang, K. Wang, Instability of standing waves of the Schrödinger equation with inhomogeneous nonlinearity, Trans. Amer. Math. Soc., 358(5) (2006), $2105-2122$.

[19] X. Liu, T. Zhang, Local well-posedness and finite time blowup for fourth-order Schrödinger equation with complex coefficient, (2020) arXiv:2010.11055v2.

[20] X. Liu, T. Zhang, Global solutions for $H^{s}$-critical nonlinear biharmonic Schrödinger equation, (2021) arXiv:2103.04293. 
[21] Y.V. Kartashov, B.A. Malomed, V.A. Vysloukh, M.R. Belic, L. Torner, Rotating vortex clusters in media with inhomogeneous defocusing nonlinearity, Opt. Lett., 42 3 (2017), 446-449.

[22] V. I. Karpman, Lyapunov approach to the soliton stability in highly dispersive systems I.Fourth order nonlinear Schrödinger equations, Phys. Lett. A, 215 (1996), $254-256$.

[23] V. I. Karpman, A. G. Shagalov, Stability of solitons described by nonlinear Schrödinger-type equations with higher-order dispersion, Phys. D, 144 (2000), 194210 .

[24] J. Kim, Y. Lee, Y. Seo, On well-posedness for the inhomogeneous nonlinear Schrödinger equation in the critical case, J. Differential Equations, 280 (2021), 179202.

[25] C.S. Liu, V.K. Tripathi, Laser guiding in an axially nonuniform plasma channel, Phys. Plasmas, 1(9) (1994), 3100-3103.

[26] F. Merle, Nonexistence of minimal blow-up solutions of equations $i u_{t}=-\Delta u-$ $k(x)|u|^{4 / N} u$ in $\mathbb{R}^{N}$, Ann. Inst. H. Poincaré Phys. Théor., 64(1) (1996), 33-85.

[27] C. Miao, G. Xu, L. Zhao, Global well-posedness and scattering for the focusing energy-critical nonlinear Schrödinger equations of fourth order in the radial case, $J$. Differential Equations, 246 (2009), 3715-3749.

[28] C. Miao, G. Xu, L. Zhao, Global well-posedness and scattering for the focusing energy critical nonlinear Schrödinger equations of fourth order in dimensions $d \geq 9$, J. Differential Equations, 251 (2011), 3381-3402.

[29] M. Nakamura, T. Wada, Modified Strichartz estimates with an application to the critical nonlinear Schrödinger equation, Nonlinear Anal., 130 (2016), 138-156.

[30] B. Pausader, Global well-posedness for energy-critical fourth-order Schrödinger equation in the radial case Dyn. Partial Differ. Equ., 4 (2007), no. 3, 197-225.

[31] B. Pausader, The focusing energy-critical fourth-order Schrödinger equation with radial data, Discrete Contin. Dyn. Syst., 24 (2009), 1275-1294.

[32] H. Pecher, Solutions of semilinear Schrödinger equations in $H^{s}$, Ann. Inst. $H$. Poincareé Phys. Théor., 67 (1997), 259-296.

[33] P. Raphaël, J. Szeftel, Existence and uniqueness of minimal blow-up solutions to an inhomogeneous mass critical NLS, J. Amer. Math. Soc., 24 (2) (2011), 471-546.

[34] I. Towers, B. A. Malomed, Stable (2+1)-dimensional solitons in a layered medium with sign-alternating Kerr nonlinearity, J. Opt. Soc. Amer. B Opt. Phys., 19 (2002), $537-543$.

[35] M. Weinstein, Nonlinear Schrödinger equations and sharp interpolation estimates, Comm. Math. Phys., 87 (1983), 567-576. 